\title{
Voluntary Task Switching: Chasing the Elusive Homunculus
}

\author{
Catherine M. Arrington and Gordon D. Logan \\ Vanderbilt University
}

\begin{abstract}
In the voluntary task switching procedure, subjects choose the task to perform on a series of bivalent stimuli, requiring top-down control of task switching. Experiments 1-3 contrasted voluntary task switching and explicit task cuing. Choice behavior showed small, inconsistent effects of external stimulus characteristics, supporting the assumption of top-down control of task choice. Switch costs were smaller when subjects chose to switch tasks than when instructed by an external cue. Experiments 4-6 separated choice costs from switch costs. These findings support models of task switching that incorporate top-down processes in accounts of switch costs. The degree to which task switching procedures capture top-down versus bottom-up processes may depend on the extent of environmental support provided by the procedure.
\end{abstract}

Keywords: voluntary task switching, executive control

Human behavior is a complex combination of actions elicited by external stimuli and emitted under voluntary control of the individual. The interaction of elicited bottom-up and emitted top-down influences on human behavior can be seen in task switching experiments, in which responses to the same set of stimuli differ depending on the goals of the individual at any point in time. Switching from one task to another produces costs in response time and accuracy, and the interpretation of these switch costs has become controversial. Some researchers attribute them to topdown influences; other researchers attribute them to bottom-up influences. In the current research, we take a novel approach to addressing this controversy using a new voluntary task switching procedure (Arrington \& Logan, 2004a). Unlike other task switching procedures, voluntary task switching requires subjects to choose the task to be performed on a given trial and thus ensures that a top-down act of control is involved in task switching. The voluntary task switching procedure inverts the usual question in task switching experiments. Instead of asking whether switch costs reflect a top-down act of control, it asks whether a top-down act of control produces switch costs. We report the results of six experiments examining switch costs in the voluntary task switching procedure, which provide strong evidence that the answer to this question is "yes." This research shifts the balance of evidence toward models of task switching that invoke top-down mechanisms in accounting for switch costs.

This research was supported by National Institute of Mental Health Grant 5F32MH069046-02 (to Catherine M. Arrington) and National Science Foundation Grants BCS 0133202 and BCS 0218507 (to Gordon D. Logan). We thank Rich Carlson, Alice Healy, and an anonymous reviewer for their helpful comments on a draft of this article.

Correspondence concerning this article should be addressed to Catherine M. Arrington or to Gordon D. Logan, Department of Psychology, Vanderbilt University, 111 21st Avenue South, Nashville, TN 37203. E-mail: kate.arrington@lehigh.edu or gordon.logan@vanderbilt.edu

\section{Task Switching}

In all task switching paradigms, subjects perform two or more tasks interleaved in time. Most often, the subordinate level tasks are simple identification or categorization tasks involving digits, letters, words, or objects, in which the stimuli afford multiple tasks and the responses are punctate with reaction time (RT) as the primary measure of performance (for reviews, see Logan, 2003; Monsell, 2003). The various subordinate-level tasks may differ from each other in a number of ways, including the following: (a) perceptual aspects of the stimulus that must be attended to categorize the stimulus (Arrington, Altmann, \& Carr, 2003; Hübner, Futterer, \& Steinhauser, 2001), (b) dimensions on which categorization occurs (Meiran, 1996), (c) stimulus-response (S-R) mappings (Kleinsorge \& Heuer, 1999; Mayr, 2002), and (d) response modality (Arrington et al., 2003). A number of task switching procedures have been developed that differ in terms of how the task to be performed on a given trial is specified. The tasks may occur in an order specified at the start of a block of trials (alternating tasks: Allport, Styles, \& Hsieh, 1994; alternating runs: Rogers \& Monsell, 1995; task span: Logan, 2004) or in a random order with a cue that occurs on every trial (explicit task cuing: Meiran, 1996) or before a series of trials (extended runs: Gopher, Armony, \& Greenshpan, 2000; for a review of all procedures, see Monsell, 2003). Robust switch costs are generally found in all of these procedures.

Explanations of switch costs fall into two basic categories: top-down accounts, which propose endogenous executive processes that actively configure the cognitive system to perform a given task, and bottom-up accounts, which propose passive interactions between successive tasks. The contrast between top-down and bottom-up accounts has been a central and driving distinction in the theoretical work on task switching since the early and influential works of Allport et al. (1994) and Rogers and Monsell (1995). Top-down accounts claim that switch costs reflect the time required to carry out an endogenous act of control that prepares for the upcoming task by reconfiguring the subordinate processes required to perform the subordinate-level task. This act of control 
is required on switch trials but not on repetition trials, so RTs are longer on switch trials. Top-down accounts typically focus on the processes that enable a new configuration of subordinate processes (or task set), although active inhibition of abandoned task sets has also been proposed (Dreisbach, Haider, \& Kluwe, 2002; Mayr, 2002; Mayr \& Keele, 2000). The enabling processes may involve updating goals in working memory (Sohn \& Anderson, 2001), retrieving S-R mappings from long-term memory (Mayr \& Kliegl, 2000, 2003; Rubinstein, Meyer, \& Evans, 2001), or adjusting attentional biases and priorities (Arrington, 2002; Logan \& Gordon, 2001; Meiran, 2000). Top-down accounts draw support from evidence that switch costs decrease with foreknowledge of the upcoming task (Ruthruff, Remington, \& Johnston, 2001; Sohn \& Anderson, 2001) and decrease with increasing preparation intervals (Meiran, 1996; Rogers \& Monsell, 1995), suggesting that the extra endogenous act of control that occurs on switch trials can be initiated, and at least partially carried out, prior to the onset of the target stimulus.

Bottom-up accounts claim that switch costs arise from factors other than active preparation for the upcoming task. These accounts focus on passive interactions that occur between successively activated task sets. The preceding task set may interfere with performance on switch trials because it is different from the required task set, or the preceding task set may facilitate performance on repetition trials because it is the same as the required task set. As with top-down accounts, a variety of bottom-up mechanisms have been proposed to account for switch costs, including the following: (a) interference from residual activation of recently used task sets (Allport et al., 1994), (b) stimulus-primed retrieval of a previous task set by the stimulus in the current trial (Allport \& Wylie, 2000; Waszak, Hommel, \& Allport, 2003; Wylie \& Allport, 2000), (c) priming of performance when a task is repeated (Ruthruff et al., 2001; Sohn \& Carlson, 2000), or (d) priming of components of performance when a task is repeated (Arrington \& Logan, 2004b; Logan \& Bundesen, 2003, 2004; Mayr \& Kliegl, 2003). Common to these bottom-up accounts is the idea that switch costs may be explained without invoking any active control processes.

The controversy over top-down and bottom-up accounts leaves us in a quandary: The observed switch costs are consistent with the idea that they are caused entirely by top-down processes; they are consistent with the idea that they are caused entirely by bottom-up processes; and they are consistent with the idea that they are caused partly by top-down processes and partly by bottom-up processes. Thus, we cannot determine whether top-down processes cause switch costs merely from the observation of switch costs. Our goal in the current experiments was to engage top-down processes by requiring subjects to choose to switch tasks voluntarily and determine whether those top-down processes would produce switch costs. Evidence of switch costs in the voluntary task switching procedure would indicate that top-down processes can cause switch costs. Failure to find switch costs in the voluntary task switching procedure would suggest that top-down processes do not cause switch costs. Thus, the voluntary task switching procedure provides a way out of the quandary. To foreshadow our results, we found that the voluntary task switching procedure resulted in robust switch costs across all six experiments. Moreover, these switch costs varied in magnitude and time course from those measured in explicit task cuing conditions, suggesting that switch costs measured under different task switching conditions may have different underlying causes.

\section{Voluntary Task Switching}

In the voluntary task switching procedure, subjects view a series of stimuli on which two or more tasks can be performed and choose which task to perform on each stimulus. Subjects are instructed to perform each task equally often and in a random sequence. Because no external cue is provided indicating the task to be performed, subjects cannot rely entirely on bottom-up processing (i.e., on responses elicited by external stimuli). The requirement to choose which task to perform on each trial ensures that a top-down act of control is necessary to switch tasks.

Arrington and Logan (2004a) examined voluntary task switching in an experiment in which subjects saw single digits on which they performed magnitude (i.e., less than or greater than five) or parity (i.e., even or odd) judgments. The experiment showed that voluntary task switching produced large costs that decreased with increasing time between trials (from $310 \mathrm{~ms}$ to $94 \mathrm{~ms}$ for response to stimulus intervals [RSIs] of 100 and 1,000 ms, respectively). Although these switch costs appear similar to those seen in other task switching procedures, the switch costs may not result from the same underlying mechanisms. Some bottom-up interpretations applicable to other task switching procedures can be ruled out, such as priming from repeated cues (Logan \& Bundesen, 2003), because no cues were presented. In the current research, Experiments 1-3 make a direct comparison of voluntary task switching and explicit task cuing procedures and provide evidence that the resulting switch costs may indeed result from different underlying mechanisms. Experiments 4-6 present further developments of the voluntary task switching procedure to directly assess alternative top-down explanations of the switch costs found in this procedure. The switch costs may reflect the time required to carry out a reconfiguration of task set following the choice to switch tasks. Alternatively, they may reflect the time required to make the choice to switch tasks.

In the voluntary task switching procedure, the task that subjects choose becomes a dependent variable of interest in addition to RT and accuracy. Choice probability data provide insight into how subjects choose which task to perform and decide whether to switch or repeat tasks, providing a perspective on the balance between top-down and bottom-up processing that is not captured by switch cost measures. Arrington and Logan (2004a) found that subjects chose to repeat tasks more often than to switch tasks. The repetition probability was 0.678 when the time interval between trials was $100 \mathrm{~ms}$ and 0.595 when it was $1,000 \mathrm{~ms}$. Deviations from chance in random generation experiments are well documented (Nickerson, 2002; Rapoport \& Budescu, 1997), but they typically take the form of an alternation bias, opposite to the repetition bias in the voluntary task switching procedure. Open questions remain about this repetition bias and the influence of external stimuli on the choice behavior. The stimulus displays in Arrington and Logan's (2004a) study were extremely sparse, consisting of the single target digit presented on a blank screen. Experiments 1-3 introduce variability in the stimuli external to the task to examine whether subjects will allow stimuli in the task environment to drive the choice process. 


\section{Experiment 1}

We addressed two basic questions in Experiment 1. First, how do the switch costs in the voluntary task switching procedure compare with those in other task switching procedures? The explicit task cuing procedure is an appropriate candidate for comparison with the voluntary task switching procedure. In both procedures, tasks occur in a random sequence. In explicit task cuing, the task is specified by an external cue, whereas in voluntary task switching, the task is chosen by the subject. The comparison thus allows a contrast between behaviors that are elicited in a bottom-up fashion and behaviors that are emitted in a top-down fashion. In the explicit task cuing procedure, subjects view a target stimulus and a cue that indicates which task to perform on each trial. The tasks occur in a random sequence so subjects do not know the identity of the upcoming task until the cue is provided. Because of this uncertainty, the experimenter is able to control the time interval between cue and target stimuli (the stimulus onset asynchrony [SOA]) during which subjects are able to prepare for the upcoming task. Typically, switch costs decrease with increases in the interval. Thus, this procedure provides some of the clearest evidence favoring active reconfiguration accounts of switch costs (Meiran, 1996). However, recent examinations of the explicit task cuing procedure have shown that the switch costs it measures may result from bottom-up processes involved in encoding the cue rather than from top-down processes involved in switching task set (Arrington \& Logan, 2004b; Logan \& Bundesen, 2003, 2004; also see Mayr \& Kliegl, 2003).

Second, we asked whether subjects' choices are emitted, as top-down accounts suggest, or elicited by aspects of the task environment, as bottom-up accounts suggest. To address this question, we examined the factors that influenced choice. We analyzed the proportion of trials on which each task was performed and the proportion of trials on which task repetitions and task alternations were performed as a function of factors internal and external to the task. The voluntary task switching procedure was modified to include a warning box that could serve as a cue indicating which task to perform in the explicit task cuing procedure. However, the warning box may influence choice behavior in the voluntary task switching procedure. Experiment 1 asked whether it did. There were three conditions: (a) a voluntary task switching black warning box (VTS-B) condition, in which the warning box was always black and the subject chose the task to perform on each trial, (b) a voluntary task switching colored warning box (VTS-C) condition, in which the warning box was randomly red or blue and the subject chose the task to perform, and (c) an explicit task cuing (CUE) condition, in which the warning box was randomly red or blue and the color of the warning box indicated which task to perform. The VTS-B condition was presented first to establish a baseline for task choice behavior. The VTS-C condition was presented next. Comparing these two conditions allowed us to examine whether the introduction of a variable external stimulus affected choice behavior. Finally, the CUE condition was presented. This condition occurred last to avoid any potential carryover of associations formed between warning box colors and tasks in the explicit task cuing condition to the voluntary task switching conditions.

\section{Method}

Subjects. Seventeen subjects, recruited from the Vanderbilt University (Nashville, Tennessee) community, participated in exchange for course credit or for $\$ 10$. Subjects reported normal or corrected-to-normal vision and were naïve to the purposes of the experiment. Data from one subject were excluded from analyses because of a failure to produce an adequate number of task switches in the voluntary task switching procedures (proportion of task repetitions for this subject was 0.88 ).

Tasks: Subordinate-level tasks and switching procedures. The subordinate-level tasks were two number judgment tasks performed on single digits: magnitude (i.e., less than or greater than five) and parity (i.e., even or odd). In the explicit task cuing procedure, the color of a warning box in the trial display indicated the task to be performed on that trial. In the voluntary task switching procedure, the subjects were instructed to choose a task to perform on each trial, trying to perform each task equally often in a random sequence. Subjects were given the following instructions:

"Now that you have practiced each task individually, you will do blocks of trials where you will be mixing together these two tasks within a single block of trials. You only need to do one task on each trial—press one button for each digit you see. You have to choose which task to perform on each trial. You should perform each task on about half of the trials and should perform the tasks in a random order. For example imagine that you had a coin that said Even-Odd on one side and Low-High on the other. Try to perform the tasks as if flipping the coin decided which task to perform. So sometimes you will be repeating the same task and sometimes you will be switching tasks. We do not want you to count the number of times you have done each task or alternate strictly between tasks to be sure you do each one half the time. Just try to do them randomly."

Design. The first manipulated variable was switching procedure. The three task switching procedures were as follows: VTS-B, VTS-C, and CUE. The second manipulated variable was the SOA between the warning box and the target: 0,300,600, and $900 \mathrm{~ms}$. Trials were assigned post hoc to task repetition, and task alternation conditions on the basis of the tasks that were performed on trial $n$ and trial $n-1$ for the VTS-B and VTS-C conditions and on the cue on trial $n$ and trial $n-1$ for the CUE condition.

Apparatus and stimuli. The presentation of stimuli and recording of responses were controlled by E-Prime 1.1 software (Psychology Software Tools, 2000) that was run on a personal computer with a 19-in. $(48.26 \mathrm{~cm})$ monitor. Responses were made on a standard keyboard with the $d, f, j$, and $k$ keys. Response mappings were organized such that each task was mapped to the index and middle fingers of the same hand, with half of the subjects using the left hand for the parity task and the right hand for the magnitude task and vice versa for the other half of the subjects. The target stimuli were the digits $1,2,3,4,6,7,8$, and 9 . The digits were presented in black and were $7 \mathrm{~mm} \times 5 \mathrm{~mm}$. The targets appeared centered within square warning boxes that were $28 \mathrm{~mm} \times 28 \mathrm{~mm}$ and constructed by line segments $1 \mathrm{~mm}$ thick. The color of the warning boxes varied by condition. The color of the warning box was black in the VTS-B condition and either red or blue in the VTS-C and CUE conditions. In the CUE condition, half the subjects performed the parity task for blue warning boxes and the magnitude task for red warning boxes, and vice versa for the other half of the subjects. All stimuli were displayed on a white background.

Procedure. The experiment began with written and verbal instructions and practice trials for each of the subordinate level tasks to familiarize the subjects with the S-R mappings for each task. Following individual task practice, subjects received instructions for the first of the task switching procedures. The three switching procedures were presented in a sequential fashion, with all subjects receiving the procedures in the same order: VTS-B, VTS-C, and CUE. For each switching procedure, subjects completed eight blocks of 64 trials each. Each of the eight target stimuli was presented with each warning box color and SOA combination. The trial order was randomized within the block. Trials began with the onset of the warning box, followed by the target digit after a $0-, 300-, 600-$, or $900-\mathrm{ms}$ 
SOA. The warning box and target remained on the screen until a response was made, and then the screen cleared for a 100-ms response to cue interval.

Data processing. In the voluntary task switching conditions, we categorized the task subjects performed on the basis of the hand they used to respond on each trial. Error trials were categorized on the basis of the hand subjects used rather than the finger used, on the assumption that subjects used the correct hand but wrong finger for the intended task (Miller, 1982; Rosenbaum, Kenny, \& Derr, 1983; Scheffers \& Coles, 2000). After task labels were assigned to trials, the trials were categorized into task repetitions and task alternations on the basis of the task performed on trial $n$ and trial $n-1$. For voluntary task switching conditions, calculations of choice probabilities included all trials except the first trial of each block. For both voluntary and cued task switching conditions, calculations of mean RTs excluded the first trial of each block, error trials, and trials with RTs less than $150 \mathrm{~ms}$ or greater than $3,000 \mathrm{~ms}$. Data from individual subjects were excluded from analyses if either response accuracy was below $90 \%$ or the task repetition probability was above 0.8 in the voluntary task switching procedure.

The choice probability data provide information about the tasks and task transitions that subjects choose to perform in the voluntary task switching conditions. To examine the factors that might influence the choice of task and choice to switch or repeat tasks, we considered the choice probabilities in two ways. First, at a group level, mean repetition probability was calculated for the conditions of interest by averaging the repetition probability for individual subjects. The mean probability values were compared with the repetition probability on the basis of chance (0.5) by constructing 95\% confidence intervals around the mean probability for the group (i.e., 1.96 times the estimated standard error of the mean for the probability). Differences in the mean probabilities between two or more conditions were assessed with analysis of variance (ANOVA), with an alpha level for significance testing set at .05 . Second, the choice probability data were also analyzed at the level of individual subjects. We conducted contingency analyses for individual subjects using chi-square statistics, with an alpha level for significance testing set at .05 . In most cases, these contingency analyses were run to rule out the impact of a particular factor on choice probability, so we did not make adjustments for multiple comparisons to increase the chances of finding a contingency between factors and choice behavior if one existed.

\section{Results and Discussion}

Choice probability analyses. The choice probability analyses focused on factors that affected the choices made by subjects to perform specific tasks and to repeat or switch tasks. These analyses addressed the question of whether subjects emit choices or let their choices be elicited by external stimuli. The weaker the relation between external stimulus variation and task choice, the greater the evidence that the choice behavior is voluntary.

The first group of analyses looked at factors that might affect the choice of the task to perform on a given trial. Subjects were instructed to perform each task on half of the trials. Subjects complied fairly well with these instructions, performing the parity task with a probability of 0.515 in the VTS-B condition and 0.509 in the VTS-C condition. The value of 0.5 that would be expected if tasks were performed equally often fell within the $95 \%$ confidence intervals for the group mean for the VTS-C condition but not the VTS-B condition.

Although subjects performed each task on approximately half of the trials, it is possible that choice probability was a function of the identity of the target digit on a given trial (e.g., magnitude judgments might be performed more frequently for digits further from five). Table 1 shows the proportion of trials on which the parity task was performed as a function of the target digit, averaged across subjects. There was little difference in the likelihood that a particular target digit was associated with one task or the other. There was no significant effect of target digit on choice probability, $F(7,105)<1$. However, at the level of individual subjects, 5 of 16 subjects showed a significant relationship between specific target digits and the task being performed. These effects were unsystematic both across subjects and within individual subjects.

Of primary interest in these analyses was whether the color of the warning box affected which task subjects choose to perform in the VTS-C condition. To address this question, we looked at the likelihood of choosing a given task as a function of the color of the warning box. The difference in probability for performing a par-

Table 1

Proportion of Trials on Which the Parity Task Was Performed as a Function of the Target Identity

\begin{tabular}{|c|c|c|c|c|c|c|c|c|}
\hline \multirow[b]{2}{*}{ Trial } & \multicolumn{8}{|c|}{ Target } \\
\hline & 1 & 2 & 3 & 4 & 6 & 7 & 8 & 9 \\
\hline \multicolumn{9}{|c|}{ Experiment 1} \\
\hline M & 0.495 & 0.512 & 0.519 & 0.518 & 0.523 & 0.506 & 0.515 & 0.509 \\
\hline SEM & 0.021 & 0.015 & 0.019 & 0.014 & 0.017 & 0.013 & 0.020 & 0.018 \\
\hline \multicolumn{9}{|c|}{ Experiment 2} \\
\hline$M$ & 0.513 & 0.547 & 0.489 & 0.525 & 0.518 & 0.503 & 0.503 & 0.448 \\
\hline$S E M$ & 0.016 & 0.014 & 0.014 & 0.018 & 0.018 & 0.015 & 0.014 & 0.019 \\
\hline \multicolumn{9}{|c|}{ Experiment 3} \\
\hline M & 0.468 & 0.537 & 0.499 & 0.526 & 0.541 & 0.494 & 0.526 & 0.517 \\
\hline$S E M$ & 0.020 & 0.018 & 0.011 & 0.014 & 0.009 & 0.017 & 0.012 & 0.025 \\
\hline \multicolumn{9}{|c|}{ Experiment 4} \\
\hline$M$ & 0.488 & 0.536 & 0.501 & 0.514 & 0.523 & 0.493 & 0.495 & 0.510 \\
\hline$S E M$ & 0.019 & 0.015 & 0.018 & 0.009 & 0.013 & 0.012 & 0.014 & 0.014 \\
\hline \multicolumn{9}{|c|}{ Experiment 5} \\
\hline M & 0.478 & 0.511 & 0.520 & 0.516 & 0.495 & 0.521 & 0.529 & 0.504 \\
\hline SEM & 0.016 & 0.017 & 0.010 & 0.014 & 0.017 & 0.016 & 0.015 & 0.015 \\
\hline
\end{tabular}


ticular task for the color for which it was more likely and the color for which it was less likely averaged 0.046 (range $=0.008-$ 0.115). At the level of individual subjects, 2 of 16 subjects showed a significant relationship between the color of the warning box and the task being performed. These analyses suggest that on the vast majority of trials for all of the subjects, the choice of task was not influenced by the color of the warning box.

The second group of analyses looked at the transition conditions-task repetitions versus task alternations-rather than specific tasks performed on a given trial. If the choice of task was truly random, then the probability of a task repetition should be 0.5 . The proportion of task repetitions are shown in Table 2 as a function of SOA, separated for the VTS-B and VTS-C conditions. Averaged over SOA, the mean repetition probability was 0.558 for the VTS-B condition and 0.577 for the VTS-C condition, replicating the repetition bias seen in Arrington and Logan's (2004a)

Table 2

Proportion of Task Repetitions as a Function of Trial Timing

\begin{tabular}{|c|c|c|c|c|}
\hline \multirow[b]{2}{*}{ Trial } & \multicolumn{4}{|c|}{ Timing interval $^{\mathrm{a}}$} \\
\hline & 0 & 300 & 600 & 900 \\
\hline \multicolumn{5}{|c|}{ Experiment 1} \\
\hline \multicolumn{5}{|c|}{ VTS-B } \\
\hline M & 0.610 & 0.565 & 0.526 & 0.531 \\
\hline SEM & 0.033 & 0.030 & 0.031 & $0.02 \mathrm{C}$ \\
\hline \multicolumn{5}{|l|}{ VTS-C } \\
\hline$M$ & 0.626 & 0.593 & 0.551 & 0.537 \\
\hline SEM & 0.038 & 0.037 & 0.041 & 0.036 \\
\hline \multicolumn{5}{|c|}{ Experiment 2} \\
\hline \multicolumn{5}{|c|}{ CUE-VTS } \\
\hline$M$ & 0.586 & 0.543 & 0.515 & $0.51 c$ \\
\hline SEM & 0.035 & 0.035 & 0.030 & 0.028 \\
\hline \multicolumn{5}{|c|}{ VTS-CUE } \\
\hline$M$ & 0.590 & 0.574 & 0.556 & 0.543 \\
\hline SEM & 0.033 & 0.031 & 0.022 & 0.024 \\
\hline \multicolumn{5}{|c|}{ Experiment 3} \\
\hline \multicolumn{5}{|c|}{ CUE-VTS } \\
\hline$M$ & 0.568 & 0.542 & 0.535 & 0.502 \\
\hline SEM & 0.033 & 0.037 & 0.031 & 0.026 \\
\hline \multicolumn{5}{|c|}{ VTS-CUE } \\
\hline$M$ & 0.631 & 0.620 & 0.556 & 0.563 \\
\hline SEM & 0.022 & 0.021 & 0.023 & 0.020 \\
\hline \multicolumn{5}{|c|}{ Experiment 4} \\
\hline \multicolumn{5}{|c|}{ Pairs } \\
\hline$M$ & 0.642 & 0.623 & 0.590 & 0.544 \\
\hline SEM & 0.040 & 0.045 & 0.040 & 0.043 \\
\hline \multicolumn{5}{|l|}{ Triplets } \\
\hline$M$ & 0.595 & 0.565 & 0.503 & 0.488 \\
\hline SEM & 0.029 & 0.031 & 0.033 & 0.031 \\
\hline \multicolumn{5}{|c|}{ Experiment 5} \\
\hline M & 0.560 & 0.550 & 0.507 & 0.497 \\
\hline SEM & 0.024 & 0.031 & 0.035 & 0.025 \\
\hline \multicolumn{5}{|c|}{ Experiment 6} \\
\hline M & 0.578 & 0.569 & 0.547 & 0.540 \\
\hline SEM & 0.025 & 0.027 & 0.028 & 0.027 \\
\hline
\end{tabular}

Note. $\quad$ VTS-B = voluntary task switching black warning box condition; VTS-C = voluntary task switching colored warning box condition; CUEVTS $=$ explicit task cving-voluntary task switching condition; VTSCUE $=$ voluntary task switching-explicit task cving condition.

a The timing interval in milliseconds represents the stimulus onset asynchrony between cue and target stimuli for Experiments 1-3, the response to stimulus intervals for Experiment 4, and the response to stimulus intervals minus $100 \mathrm{~ms}$ for Experiments 5 and 6. study. For both VTS-B and VTS-C conditions, the chance probability of a repetition fell below the $95 \%$ confidence interval of the mean probabilities for the 0 - and 300-ms SOA conditions but not for the 600- and 900-ms conditions. The time course of the repetition bias was examined in a 2 (switching procedure: VTS-B and VTS-C) $\times 4$ (SOA: 0, 300, 600, and $900 \mathrm{~ms}$ ) repeated measures ANOVA on the repetition probability. The main effect of switching procedure was not significant, $F(1,15)=1.7, p>.20$, $M S E=0.0066$, indicating that repetition probability was not different for black versus colored warning boxes. The main effect of SOA was significant, $F(3,45)=17.2, p<.01, M S E=0.0029$. Pairwise comparisons showed that repetition probability was significantly greater at the 0-ms SOA than at the 300-ms SOA, which was significantly greater than repetition probability at the $600-\mathrm{ms}$ or 900-ms SOAs, which did not differ from each other.

The subjects' choice to repeat or switch tasks might be affected by a number of different factors. In the current study, we were interested in whether the color of the warning box in the VTS-C condition influenced the choice. We examined repetition probability as a function of a change in color of the warning box (i.e., whether subjects were more likely to repeat the task from the previous trial if the warning box color repeated). The repetition probabilities when the warning box repeated versus changed colors are shown in Table 3. The difference between these conditions (0.016) failed to reach significance, $F(1,15)=3.5, p=.08$, $M S E=0.0006$. At the level of individual subjects, 0 of 16 subjects showed a significant relationship between repetition of warning box color and task repetition probability. Taken together with the analyses above showing that choice probabilities did not differ for black and colored warning boxes, these analyses provide strong evidence that subjects emitted their choices and did not allow them to be elicited by unrelated information in the environment.

Aspects of the target stimuli may also affect subjects' choice to repeat or switch tasks. Two possibilities come to mind. First, subjects might be more likely to repeat a task if the response on trial $n-1$ would repeat on trial $n$ if the task were repeated (e.g., target stimuli " 7 " on trial $n-1$ and "9" on trial $n$ ) than if the response would switch (e.g., target stimuli "7" on trial $n-1$ and " 4 " on trial $n$ ). The mean repetition probabilities averaged across the VTS-B and VTS-C conditions for these potential response repetition and response switch trials are shown in Table 3. Across subjects, there was not a reliable difference in repetition probability for these two conditions, $F(1,15)=1.4, p>.2, M S E=$ 0.0011 . At the level of individual subjects, 4 of 16 subjects were significantly more likely to repeat the task in the case of potential response repetitions, and 1 of 16 subjects was less likely to repeat the task in the case of a potential response repetition.

Second, subjects might be more likely to repeat a task if the target digit repeats from trial $n-1$ to trial $n$. The mean repetition probabilities for target repetition and target change trials are shown in Table 3 . Across subjects, repetition probability was significantly greater for trials when the target repeated than for trials when the target changed, $F(1,15)=23.2, p<.05, M S E=0.0028$. At the level of individual subjects, 8 of 16 subjects were significantly more likely to repeat the task if the target repeated than if the target changed. Thus, subjects' choices to repeat or switch tasks were influenced to some extent by the nature of the relationship between the targets on trial $n$ and trial $n-1$. However, target repetitions 
Table 3

Proportion of Task Repetitions as a Function of Repetitions or Switches of the Warning Box Color, Potential Response on Task Repetitions, and Target

\begin{tabular}{|c|c|c|c|c|c|c|}
\hline \multirow[b]{2}{*}{ Trial } & \multicolumn{2}{|c|}{ Warning box color } & \multicolumn{2}{|c|}{ Response } & \multicolumn{2}{|c|}{ Target } \\
\hline & Repeat & Switch & Repeat & Switch & Repeat & Switch \\
\hline \multicolumn{7}{|c|}{ Experiment 1} \\
\hline M & 0.585 & 0.569 & 0.575 & 0.561 & 0.647 & 0.558 \\
\hline SEM & 0.038 & 0.034 & 0.036 & 0.029 & 0.042 & 0.031 \\
\hline \multicolumn{7}{|c|}{ Experiment 2} \\
\hline M & 0.564 & 0.543 & 0.550 & 0.556 & 0.604 & 0.548 \\
\hline$S E M$ & 0.030 & 0.026 & 0.035 & 0.022 & 0.040 & 0.026 \\
\hline \multicolumn{7}{|c|}{ Experiment 3} \\
\hline M & 0.579 & 0.561 & 0.574 & 0.555 & 0.600 & 0.561 \\
\hline SEM & 0.022 & 0.028 & 0.024 & 0.028 & 0.025 & 0.027 \\
\hline \multicolumn{7}{|c|}{ Experiment 4} \\
\hline M & & & 0.579 & 0.558 & 0.573 & 0.568 \\
\hline SEM & & & 0.034 & 0.032 & 0.032 & 0.033 \\
\hline \multicolumn{7}{|c|}{ Experiment 5} \\
\hline M & & & 0.541 & 0.528 & 0.571 & 0.529 \\
\hline SEM & & & 0.027 & 0.029 & 0.027 & 0.029 \\
\hline
\end{tabular}

were not frequent (they occurred on $1 / 8$ of the trials), so this dependency did not have a strong effect on overall performance.

$R T$ analyses. The switch cost analysis looked at the effect of task repetitions versus task alternations as a function of switching procedure and SOA, focusing on the time course of the task switching effects. Figure 1 shows the time-course data for task repetitions and task alternations for each of the three switching procedures (i.e., RT as a function of SOA). For each procedure, RT was slower on task alternations than on task repetitions, showing the expected switch costs. Switch costs showed the typical timecourse function in each procedure, decreasing monotonically with increasing SOA.

The RT data were entered into a 3 (switching procedure: VTS-B, VTS-C, and CUE) $\times 2$ (task transition: repetition and alternation $) \times 4$ (SOA: 0, 300, 600, and $900 \mathrm{~ms}$ ) repeated mea-

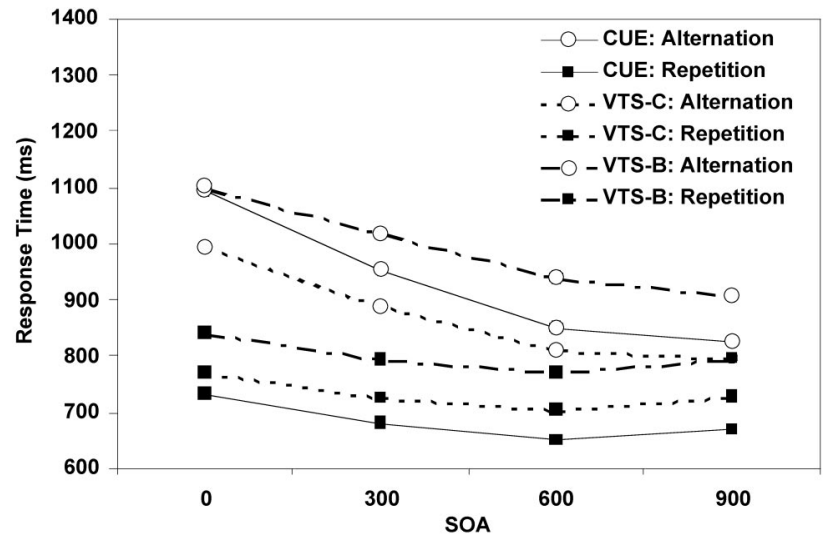

Figure 1. Experiment 1: Mean response time in milliseconds as a function of stimulus onset asynchrony (SOA) for the task repetition (solid squares) and task alternation (open circles) trials for the voluntary task switching black warning box (VTS-B; dashed/dotted lines), voluntary task switching colored warning box (VTS-C; dotted lines), and explicit task cuing (CUE; solid lines) procedures. sures ANOVA. RTs were longer in the VTS-B than in the VTS-C and CUE conditions, $F(2,30)=10.2, p<.05, M S E=34,659.6$ $(M \mathrm{~s}=895,801$, and $806 \mathrm{~ms}$, respectively). Given that the VTS-B condition was always performed first, this difference in mean RTs likely reflects a practice effect. There was a general speed up of responding over increasing $\mathrm{SOA}, F(3,45)=74.8, p<.05$, $M S E=5,189.2(M \mathrm{~s}=921,842,787$, and $786 \mathrm{~ms}$ for the $0-, 300-$, 600-, and 900-ms conditions, respectively). Task repetitions were faster than task alternations, $F(2,30)=173.9, p<.05, M S E=$ 20,418.4 (Ms $=738 \mathrm{~ms}$ and $930 \mathrm{~ms}$ ), showing the expected switch costs. The switch costs decreased monotonically over increasing SOA, $F(3,45)=36.8, p<.05, M S E=3,580.4$ (switch costs $=$ 281, 220, 158, and $110 \mathrm{~ms}$, for the 0-, 300-, 600-, and 900-ms conditions, respectively). Finally, switch costs varied across the switching procedures, $F(2,30)=9.5, p<.05, M S E=9,618.6$, with switch costs greatest in the CUE condition $(247 \mathrm{~ms})$ followed by the VTS-B and VTS-C conditions (189 ms and $140 \mathrm{~ms}$, respectively).

Two general conclusions can be drawn from the results of Experiment 1 . First, the choice probability analyses indicate that task choice is made endogenously, because choice probability was relatively uninfluenced by external stimuli. Second, the RT data show robust switch costs in both voluntary task switching procedures, demonstrating that endogenous task choices do result in switch costs. Furthermore, the switch costs in the voluntary task switching procedure were different from those in the explicit task cuing procedure. The difference in switch costs between these two procedures suggests that the switch costs may have different causes. However, the interaction with switching procedure is difficult to interpret because practice was confounded with switching procedure. Practice is not likely to account for the differences in switch costs between the CUE and VTS-C conditions because these conditions did not differ in overall RT, suggesting that practice had the largest influence on the first procedure, VTS-B. Furthermore, although switch costs decreased between the VTS-B and VTS-C conditions, as would be expected with practice, switch costs were largest for the CUE condition, which occurred last and 
would be expected to produce the smallest switch costs if differences in switch cost were due to practice.

\section{Experiment 2}

In Experiment 1, we showed that irrelevant variation in the warning box color did not affect subjects' choice probabilities in the voluntary task switching procedure. This result allowed us to compare voluntary task switching and explicitly cued task switching in the same stimulus conditions. However, Experiment 1 confounded the order in which voluntary and cued task switching occurred, making direct comparisons between conditions difficult. In Experiment 2, subjects performed both voluntary and cued task switching with colored warning boxes with the order counterbalanced. Half of the subjects performed the voluntary task switching procedure before the explicit task cuing procedure, and the other half performed the procedures in the opposite order. Presenting explicitly cued task switching before voluntary task switching allowed us to examine the impact of prior associations on choice behavior (Allport \& Wylie, 2000; Waszak et al., 2003; Wylie \& Allport, 2000; Yeung \& Monsell, 2003a). Subjects may not be able to ignore associations they acquired between warning box colors and tasks when they are instructed subsequently to voluntarily choose which task to perform. When they transfer to the voluntary task switching procedure, their choices may be influenced by the associations they learned between the tasks and the warning box colors. If they do so, then their behavior is not voluntary but rather is elicited by external stimuli. However, if these subjects do not show a relationship between the warning box color and task choice, then their behavior is emitted without regard to external stimuli.

\section{Method}

Subjects. Nineteen subjects participated in Experiment 2. Subjects were drawn from the same population as Experiment 1. Data from three subjects were excluded from analyses because of low accuracy.

Design. The first manipulated variable was task switching procedure: voluntary task switching (VTS) and explicit task cuing (CUE). The second manipulated variable was SOA: $0,300,600$, and $900 \mathrm{~ms}$. Trials were assigned to task repetition and task alternation conditions on the basis of the responses on trial $n$ and trial $n-1$ for the VTS condition and on the cues on trial $n$ and trial $n-1$ for the CUE condition.

Apparatus, stimuli, and procedure. Except where noted, details of the experimental method were the same as in Experiment 1. The color of the warning box was either blue or red. The color-to-task mapping in the CUE condition was counterbalanced across subjects. Subjects initially practiced the two subordinate level tasks separately. Subjects then received the instructions for the first switching procedure and the experimental blocks for that condition followed by instructions for the second switching procedure and experimental blocks for that condition. The two switching procedures were presented in a sequential fashion, and the order of the procedures was counterbalanced across subjects. For each switching procedure, subjects completed 10 blocks of 64 trials each. Each of the eight target stimuli was presented with each warning box color and SOA combination. The trial order was randomized within the block.

\section{Results and Discussion}

Choice probability analyses. Subjects produced each task on approximately half of the trials. The mean probability of choosing the parity task was 0.506 , which included the chance value of 0.5 in its $95 \%$ confidence interval. The mean probabilities of choosing the parity task as a function of the target digit are given in Table 1. The effect of the target digit on choice probability was significant, $F(7,105)=3.2, p<.05, M S E=0.0042$. There was a slight tendency across subjects to perform the parity task more frequently for even target digits than for odd. At the level of individual subjects, 4 of 16 subjects showed a significant relationship between the target digit and the task chosen.

Again, we asked whether the color of the warning box influenced the choice of task. To determine whether associations between cue colors and tasks in the cuing condition carried over and influenced performance in the voluntary task switching condition, we considered the probability of performing a task when the warning box color was the color associated with that task in the CUE condition for the 8 subjects who experienced the CUE-VTS order. The mean difference in the probability of performing a task with the associated versus the unassociated warning box colors was 0.01 , which included 0 in the $95 \%$ confidence intervals around the mean. At the level of individual subjects, 1 of 8 subjects was significantly more likely to choose to perform the task that had previously been associated with the warning box color. Of the 8 subjects in the VTS-CUE order, none of the subjects showed a significant contingency between warning box color and task choice.

The great majority of subjects did not show a consistent relationship between the choice of task and the color of the warning box, even when that warning box color had previously been associated with a particular task, suggesting that subjects did not use the warning box color as a basis for choosing which task to perform (cf. Allport \& Wylie, 2000; Waszak et al., 2003; Wylie \& Allport, 2000). These results support the assumption that subjects made voluntary choices about which task to perform on each trial rather than relying on external stimuli to determine the task in a bottom-up fashion. At a methodological level, the results support the use of the explicit task cuing and voluntary task switching procedures in a within-subjects design in which order is counterbalanced. Indeed, order of procedure did not interact significantly with any effects in the choice probability or RT data when examined as a between-subjects factor in the remaining analyses.

As in Experiment 1, task transitions were analyzed by examining the probability of repeating a task as a function of a number of possible factors that might influence choice behavior. The proportions of task repetitions are shown in Table 2 as a function of SOA. The mean repetition probability, averaged over SOA, was 0.553 , replicating the repetition bias seen in previous experiments. The chance probability of a repetition fell below the $95 \%$ confidence interval for the 0 -ms SOA but not for the other three conditions. The effect of SOA was examined in a 4 (SOA: 0, 300, 600, and $900 \mathrm{~ms}) \times 2$ (order: VTS-CUE and CUE-VTS) mixed measures ANOVA. Only the main effect of SOA was significant, $F(3,42)=$ $5.0, p<.05, M S E=0.0022$. Pairwise comparisons showed that the repetition probability was greater in the 0 -ms condition than in the $600-\mathrm{ms}$ or $900-\mathrm{ms}$ conditions.

Other contingencies in choice behavior were also examined. Mean probabilities for task repetitions for each of these contingencies are given in Table 3. Subjects were slightly more likely to repeat the task if the color of the warning box repeated from trial $n-1$ to trial $n, F(1,14)=4.5, p=.053, M S E=0.0008$, with 2 
of 16 subjects showing this effect significantly. Subjects were no more likely to repeat the task if there was a potential response repetition, $F(1,14)<1$; however, at the level of individual subjects, 2 of 16 subjects were significantly more likely to repeat in this situation, and 2 of 16 subjects were significantly less likely to repeat. Finally, subjects were more likely to repeat the task if the target repeated from trial $n-1$ to trial $n, F(1,14)=7.8, p<.05$, $M S E=0.0031$, with 3 of 16 subjects showing this effect, and 1 of 16 showing significantly more task repetitions when the target switched. As in the previous experiment, the choice probability data suggest that subjects emitted their choices voluntarily on the vast majority of trials rather than letting their choices be elicited by aspects of the task environment.

$R T$ analyses. The analyses of the RT data allowed us to compare switch costs in the voluntary task switching and explicit task cuing procedures without confounding practice. The data are graphed in Figure 2, which shows the time-course functions for task repetitions and task alternations for both the VTS and CUE conditions. Replicating Experiment 1, switch costs were smaller for the VTS than for the CUE condition because of slower responding on repetition trials in the VTS condition as compared with the CUE condition. Additionally, switch costs for both conditions decreased with increasing SOA.

The data were analyzed in a 2 (switching procedure: VTS and CUE) $\times 2$ (task transition: repetition and alternation) $\times 4$ (SOA: $0,300,600$, and $900 \mathrm{~ms}) \times 2$ (order: VTS-CUE and CUE-VTS) mixed factors ANOVA with order as a between-subjects factor. The effect of order was not significant and did not enter into any significant interactions. There was no overall difference in RTs between the two switching procedures, $F(1,14)=1.8, p=.20$, $M S E=367,161.2$. The main effects of task transition and SOA were both significant, $F(1,14)=112.5, p<.05, M S E=29,550.0$, and $F(3,42)=46.6, p<.05, M S E=4,837.7$, as was the interaction between these two factors, $F(3,42)=24.3, p<.05$, $M S E=2,152.6$. Critical for the comparison of switch costs in voluntary and explicitly cued task switching, the interaction between switching condition and task transition was significant, $F(1$,

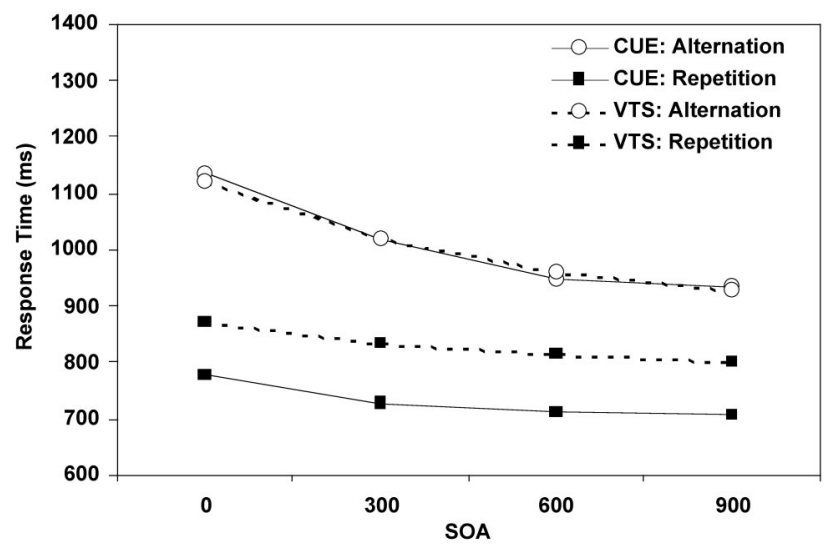

Figure 2. Experiment 2: Mean response time in milliseconds as a function of stimulus onset asynchrony (SOA) for the task repetition (solid squares) and task alternation (open circles) trials for the voluntary task switching (VTS; dotted lines), and explicit task cuing (CUE; solid lines) procedures.
14) $=18.1, p<.05, M S E=906.9$, with switch costs greater in the CUE condition (279 ms) than in the VTS condition $(177 \mathrm{~ms})$. Additionally, the three-way interaction of switching procedure, task transition, and SOA was not significant, $F(3,42)<1$, indicating that the time-course functions of the transition conditions were similar for the two switching procedures. One particular aspect of the time-course functions deserves a comment: the task alternations in both CUE and VTS conditions were virtually overlapping with all of the difference in switch costs resulting from the slower responses on task repetitions for the VTS condition. This result might suggest that task alternations are the same in the two procedures and that only task repetitions differ. However, this pattern is not repeated in either Experiments 1 or 3 and thus speculating further about this overlap appears unfounded.

Two important conclusions can be drawn from Experiment 2. First, the choice probability data demonstrated that task choice generally was unaffected by external stimuli, even when these stimuli were associated previously with particular tasks. This finding provides strong evidence that task choice is under top-down control. Second, as in Experiment 1, there were substantial switch costs that arose from voluntary task switching, replicating the findings from Arrington and Logan (2004a): An endogenous choice of the task to perform produces switch costs. Furthermore, in both Experiments 1 and 2, switch costs were substantially larger in the explicit task cuing procedure than in the voluntary task switching procedure. We address the possible causes for this difference in Experiment 3.

\section{Experiment 3}

Two interpretations of the difference in switch costs between voluntary and explicitly cued task switching may be posited. First, the underlying causes for the two switch costs may be entirely different. Second, there may be common causes for the switch costs, but the explicit task cuing procedure may involve some extra process, such that it exaggerates switch costs. A number of researchers have shown that switch costs in the explicit task cuing procedure result from a cue encoding benefit as well as or instead of endogenous processes that change task set (Arrington \& Logan, 2004b; Logan \& Bundesen, 2003, 2004; Mayr \& Klieg1, 2003). This cue encoding benefit can be demonstrated with a modified version of the explicit task cuing procedure in which two cues are used for each task. This manipulation produces three different types of task transitions: cue repetitions, task repetitions, and task alternations. Under a number of experimental conditions, researchers have found that a large portion of the switch costs results from differences between cue repetition and task repetition conditions rather than differences between task repetition and task alternation conditions (Logan \& Bundesen, 2003; Mayr \& Kliegl, 2003). The voluntary task switching procedure does not provide an external cue, so a cue encoding benefit cannot contribute to the switch costs.

Following previous researchers, we used two cues for each task to separate cue encoding benefits from switch costs. The parity and magnitude judgments from the previous experiments were cued with four different warning box colors, with two colors for each task. This procedure produces three task transitions: (a) cue repetitions, in which the cue color and the task are the same, (b) task repetitions, in which the cue color changes, but the task remains 
the same, and (c) task alternations, in which the cue color and the task both change. The cue-switch effect refers to the difference between cue repetitions and task repetitions; the task-switch effect refers to the difference between task repetitions and task alternations (Logan \& Bundesen, 2003, 2004; Mayr \& Kliegl, 2003). As in Experiment 2, the voluntary task switching procedure and the explicit task cuing procedure were presented in a within-subjects design, with the order counterbalanced. Switch costs in the voluntary task switching procedure were compared with the task-switch effect. We compared both the magnitude and the time course of the effects.

\section{Method}

Subjects. Seventeen subjects participated in Experiment 3. Data from one subject were excluded from analyses because of a failure to produce an adequate number of task switches in the voluntary task switching procedure (proportion of task repetitions for this subject was 0.83 ).

Design. The first manipulated variable was task switching procedure: voluntary task switching (VTS) and explicit task cuing (CUE). The second manipulated variable was SOA: 0, 300, 600, and $900 \mathrm{~ms}$. In the VTS condition, trials were assigned to task repetition and task alternation conditions on the basis of the responses on trial $n$ and trial $n-1$. In the CUE condition, trials were assigned to cue repetition, task repetition, and task alternation conditions on the basis of the cues presented on trial $n$ and trial $n-1$.

Apparatus, stimuli, and procedure. The apparatus and stimuli were the same as in previous experiments, except as noted. The warning box colors were red, green, blue, and yellow. Red and green were mapped to one task, and blue and yellow were mapped to the other task. Task-to-color mappings were counterbalanced across subjects. The background color was light gray. Subjects initially practiced the two subordinate level tasks separately. Subjects then received the instructions for the first switching procedure and the experimental blocks for that condition followed by instructions for the second switching procedure and experimental blocks for that condition. The two switching procedures were presented sequentially, and the order of the procedures was counterbalanced across subjects. For each switching procedure, subjects completed 10 blocks of 64 trials, with warning box color, target stimulus, and SOA selected randomly without replacement from all possible combinations of these factors.

\section{Results and Discussion}

Choice probability analyses. The mean probability of doing the parity task was 0.514 , which excluded the chance value of 0.5 in the $95 \%$ confidence interval, indicating that the parity task was performed on slightly more than half the trials. The mean probabilities of doing the parity task as a function of the target digit are given in Table 1 . The effect of the target digit on the choice of task was significant, $F(7,98)=2.4, p<.05, M S E=0.0041$. There was a slight tendency across subjects to perform the parity task more frequently for even target digits than for odd. At the level of individual subjects, 6 of 16 subjects showed a significant relationship between the target digit and the task chosen.

Again, we considered the possibility that associations between cue colors and tasks in the explicit task cuing condition may carry over and influence performance in the voluntary task switching condition by examining the probability of performing a task when the warning box color was one of the two colors associated with that task in the CUE condition for the 8 subjects who performed in the CUE-VTS order. The mean difference in the probability of performing a task with the associated versus the unassociated warning box colors was 0.01 , which included 0 in the $95 \%$ confidence interval around the mean. At the level of individual subjects, 2 of 8 subjects were significantly more likely to choose to perform the task that had not previously been associated with the warning box color. Of the 8 subjects in the VTS-CUE order, none of the subjects showed a significant contingency between warning box color and task choice. As in Experiment 2, the effect of order in which subjects performed the two switching procedures did not interact with other variables in either the choice probability or RT analyses, with one exception for the RT data, which is noted below.

Task transitions were analyzed by examining the probability of repeating a task as a function of a number of possible factors that might influence choice behavior. The proportions of task repetitions are shown in Table 2 as a function of SOA. The mean repetition probability, averaged over SOA, was 0.565 , replicating the repetition bias seen in previous experiments. The chance probability of a repetition fell below the $95 \%$ confidence interval for the 0 - and 300-ms SOAs but not for the 600- and 900-ms SOAs. The effect of SOA was examined in a 4 (SOA: 0, 300, 600, and $900 \mathrm{~ms}) \times 2$ (order: VTS-CUE and CUE-VTS) mixed measures ANOVA, with order as a between-subjects factor. The main effect of SOA was significant, $F(3,42)=7.9, p<.05$, with pairwise comparisons showing that the repetition probability was greater in the 0 -ms SOA than in the 600-ms or 900-ms SOAs and greater in the 300-ms SOA than in the 900-ms SOA.

We also considered the contingencies between choice behavior and various factors, including the following: repetition of the warning box color, potential response repetition, and target repetition. Mean probabilities for task repetitions for each of these contingencies are given in Table 3. At the group level, none of the factors had a significant influence on repetition probability: warning box color repetition, $F(1,14)=1.1, p=.31, M S E=0.0025$; potential response repetition, $F(1,14)=2.0, p=.18, M S E=$ 0.0013; and target repetition, $F(1,14)=2.7, p=.12, M S E=$ 0.0046. At the individual level, there were some significant effects: For (a) warning box color change, 1 of 16 subjects was more likely to repeat the task if the warning box color repeated; (b) potential response repetition, 1 of 16 subjects was more likely to repeat the task if there was a potential response repetition, and 1 of 16 subjects was less likely to repeat the task; and (c) target repetition, 3 of 16 subjects were more likely to repeat the task if the target repeated. As in the previous experiments, the choice probability data showed relatively weak contingencies between subjects' choices and aspects of the task environment. This supports the conclusion that subjects' choices were emitted voluntarily and not elicited by stimuli in the environment.

RT analyses. Figure 3 shows the time-course functions for task repetitions and task alternations in the VTS condition and the cue repetitions, task repetitions, and task alternations in the CUE condition. In the CUE condition, responding was fastest in the cue repetition condition, followed by the task repetition and task alternation conditions. The size of the cue-switch effect decreased with increasing SOA, whereas the task-switch effect was constant over SOA. This pattern of results is similar to that seen in other experiments that have used the four-cue/two-task procedure. The magnitude of the task-switch effect is larger than in experiments with meaningful words as cues (Arrington \& Logan, 2004b; Logan 


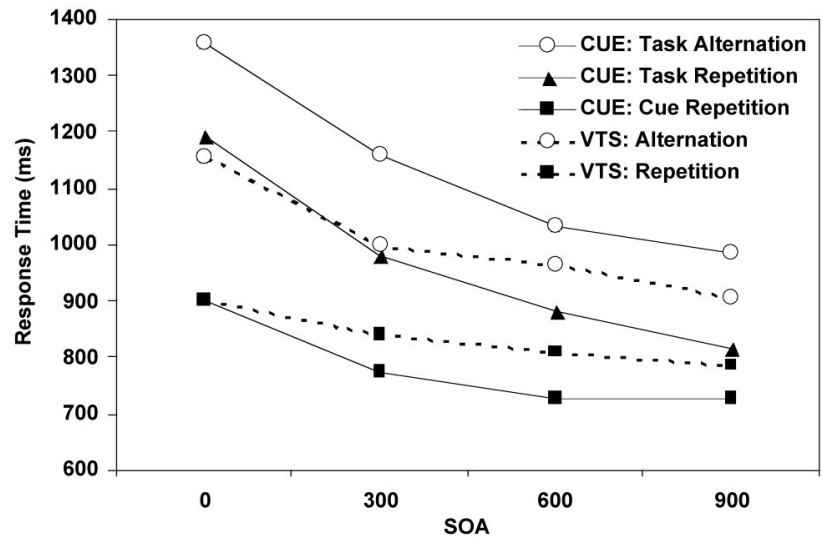

Figure 3. Experiment 3: Mean response time in milliseconds as a function of stimulus onset asynchrony (SOA) for the cue repetition (solid squares), task repetition (solid triangles), and task alternation (open circles) trials for the explicit task cuing (CUE; solid line) procedure, and task repetitions (solid squares) and task alternations (open circles) for the voluntary task switching (VTS; dotted lines) procedure.

\& Bundesen, 2003, 2004) but similar to those seen in other experiments that have also used arbitrary (nonword) cues (Logan \& Bundesen, 2004; Mayr \& Kliegl, 2003).

In the VTS condition, the data were similar to Experiments 1 and 2. Responding was faster for task repetitions than for task alternations, with the magnitude of the switch costs decreasing as a function of SOA. The comparison of interest in the current experiment is between the switch costs in the VTS procedure and the task-switch effect in the CUE procedure. If the difference in switch costs seen in Experiments 1 and 2 reflects an exaggeration of switch costs in the CUE procedure because of cue encoding processes, then switch costs should be equivalent after cue encoding benefits are accounted for. As can be seen in the graph of the data in Figure 3, the magnitudes of the switch costs in the VTS procedure and the task-switch effect in the CUE procedure are similar (167 ms and $172 \mathrm{~ms}$, respectively). However, the timecourse functions differ, suggesting different underlying causes.

The data from the CUE condition were initially analyzed in a 3 (task transition: cue repetition, task repetition, and task alternation) $\times 4$ (SOA: $0,300,600$, and $900 \mathrm{~ms}) \times 2$ (order: VTS-CUE and CUE-VTS) mixed measures ANOVA, with order as a between-subjects factor. The main effect of order was not significant, $F(1,14)<1$, and did not interact with any of the other effects. The main effect of task transition was significant, $F(2$, $28)=97.1, p<.05, M S E=20,383.4$; pairwise comparisons showed that responding was faster in the cue repetition condition $(M=723 \mathrm{~ms})$ than in the task repetition condition $(M=967 \mathrm{~ms})$, which was faster than responding in the task alternation condition ( $M=1,134 \mathrm{~ms})$. The main effect of SOA was also significant, $F(3$, $42)=88.5, p<.05, M S E=10,186.8$; pairwise comparisons showed that RTs decreased significantly with each increase in SOA $(M \mathrm{~s}=1,150,972,881$, and $843 \mathrm{~ms}$ for 0-, 300-, 600-, and 900-ms SOAs, respectively). The interaction of these two factors was also significant, $F(6,84)=8.0, p<.05, M S E=5,059.6$. The interaction was broken down to separate the time courses of the two effects. There was a significant interaction between SOA and cue repetition versus task repetition, $F(3,42)=11.3, p<.05$, $M S E=5,267.6$, with cue-switch effect decreasing monotonically with increasing SOA (mean difference $=292,205,153$, and $88 \mathrm{~ms}$ for the 0-, 300-, 600-, and 900-ms SOAs, respectively). The interaction between SOA and task repetition versus task alternation was not significant, $F(3,42)=0.2, M S E=5,522.8$, with the task-switch effect fairly constant over SOA (switch costs $=164$, 181, 153, and $171 \mathrm{~ms}$, for the 0-, 300-, 600-, and 900-ms SOAs, respectively).

The critical question addressed in Experiment 3 was whether the differences in the size of the switch costs for voluntary and explicitly cued task switching in Experiments 1 and 2 resulted from an exaggeration of the switch costs in the explicit task cuing procedure. The hypothesis tested in the following analyses was that the switch costs in the voluntary switching procedure were the same as the task-switch effect (i.e., task alternation minus task repetition). We addressed this hypothesis in a 2 (switching procedure: VTS and CUE) $\times 2$ (task transition: task repetition and task alternation) $\times 4$ (SOA: 0, 300, 600, and $900 \mathrm{ms)} \times 2$ (order: VTS-CUE and CUE-VTS) mixed measures ANOVA. The main effect of order was not significant, $F(1,14)<1$. Subjects were significantly faster in the VTS condition $(M=920 \mathrm{~ms})$ than in the CUE condition $(M=1,051 \mathrm{~ms}), F(1,14)=16.4, p<.05, M S E=$ 66,413.0; faster for task repetitions $(M=901 \mathrm{~ms})$ than for task alternations $(M=1,071 \mathrm{~ms}), F(1,14)=62.0, p<.05, M S E=$ 29,664.7; and faster at longer SOAs, $F(3,42)=152.6, p<.05$, $M S E=6,182.6$, with pairwise comparisons showing significant decreases in RT between each incremental increase in SOA $(M \mathrm{~s}=$ 1,152, 995, 923, and $874 \mathrm{~ms}$ for the 0-, 300-, 600-, and 900-ms SOAs, respectively). Critical for comparing switch costs in VTS with the task-switch effect in CUE is the interaction between task transition and switching procedure. The two-way interaction of these variables was not significant, $F(1,14)<1$, indicating that the magnitude of the switch costs in the two procedures was equivalent. However, there was a significant three-way interaction with SOA, $F(3,42)=5.5, p<.05, M S E=2,797.5$. This three-way interaction varied with the between-subjects order factor, $F(3,42)=3.8, p<.05, M S E=2,797.5$, but the higher order interaction did not alter the interpretation of the three-way interaction. Breaking down the three-way interaction and examining the switch costs as a function of SOA for each of the switching procedures individually showed that the time course of the effect differed between procedures. Switch costs varied as a function of SOA for the VTS condition, $F(3,42)=8.5, p<.05, M S E=$ 3,159.9, decreasing monotonically over increasing SOA (switch costs $=255,158,155$, and $119 \mathrm{~ms}$, for the 0-, 300-, 600-, and 900-ms SOAs, respectively). Pairwise comparisons showed that switch costs were significantly larger at the 0-ms SOA than at the other time intervals. In the CUE condition, the task-switch effect did not vary as a function of SOA, as shown in the analysis above. Planned contrasts examining the linear trend in both conditions showed a significant linear trend in the switch costs over SOA for the VTS condition, $F(1,45)=21.4, p<.05, M S E=2,797.5$, but not for the CUE condition, $F(1,45)<1$.

Thus, although the magnitude of the switch costs during the voluntary task switching procedure $(172 \mathrm{~ms})$ was similar to the magnitude of the task-switch effect in the four-cue/two-task procedure $(167 \mathrm{~ms})$, the time course of the effect differed. This result suggests that the underlying causes of the two effects are not the 
same. The finding that the task-switch effect in the four-cue/twotask procedure did not vary as a function of SOA suggests that the difference between task repetitions and task alternations does not result from a process that is carried out in response to the cue and thus is absorbed in the longer SOAs (Mayr \& Kliegl, 2003). The constant task-switch effect across SOA is suggestive of a process that occurs following the onset of the target stimulus, like a residual switch cost. However the switch costs in the voluntary task switching procedure do show a time-course function that decreases with increasing SOA, a signature for processes that can be carried out prior to target onset (Meiran, 1996). These results are consistent with the idea that voluntary switch costs reflect top-down processing, whereas explicitly cued switch costs reflect bottom-up processing.

\section{Voluntary Task Switching Versus Explicit Task Cuing}

Experiments 1-3 compared subjects' performance in voluntary task switching and explicit task cuing procedures. Although switch costs were robust in both procedures, the magnitude of the switch costs was smaller in the voluntary task switching procedure than in the explicit task cuing procedure. This difference may reflect different underlying cognitive processes: top-down processes with voluntary task switching and bottom-up processes with explicitly cued task switching. We examined individual differences in switch costs in the two procedures. We calculated switch costs averaged across SOA for each procedure for each of the 48 subjects in Experiments 1-3. In calculating the voluntary switch costs in Experiment 1, we used only the data from the VTS-C condition to minimize the potential practice effects seen in the VTS-B condition. In calculating the explicit task cuing switch costs in Experiment 3 , we used the cue repetition and task alternation conditions, which represent the standard measure of switch costs in the explicit task cuing procedure. We calculated split-half reliability for the switch cost measures for each procedure by calculating the switch cost for even and odd blocks of trials for each subject. The split-half reliability (adjusted by the Spearman-Brown prophecy formula) for the voluntary task switching conditions was .86 and for the explicit task cuing conditions was .87. The correlation between voluntary and cued switch costs was weak but significant $(r=.36, p<.05)$; however, when the overall RT was controlled, the correlation decreased and was no longer significant $(r=.19$, $p>.2$ ). The null correlation supports the conclusion that the switch costs in voluntary task switching and explicit task cuing procedures may stem from different underlying mechanisms: topdown and bottom-up processes, respectively.

There has been a considerable amount of theoretical work aimed at understanding the cognitive processes involved in the explicit task cuing procedure (Arrington \& Logan, 2004b; Logan \& Bundesen, 2003, 2004; Mayr \& Kliegl, 2003; Schneider \& Logan, in press). The modal interpretation is that switch costs represent top-down control processes engaged in reconfiguring the cognitive system to perform a new task. On the contrary, the four-cue/twotask procedure demonstrates that a substantial proportion of the switch costs arise from bottom-up cue encoding processes. After cue encoding effects have been removed, the remaining switch costs have been variable, and the explanation of these costs is still at issue. The remaining switch costs are substantial in some experiments, as in the current Experiment 3 (also see Mayr \& Kliegl,
2003), and negligible in other experiments (Logan \& Bundesen, 2003, 2004). Mayr and Kliegl (2003) suggested that the remaining switch costs reflect the time involved in applying mapping rules to the targets. They argued that these processes are necessary for switching tasks and represent actual switch costs. However, Logan and Bundesen (2004) proposed that these remaining switch costs reflect retrieval of the task name as a mediator that is combined with the target stimulus to serve as a compound retrieval cue that pulls the response from memory (also see Emerson \& Miyake, 2003). Retrieval of the mediator is fast on task repetition trials, where it benefits from the presence of the previous mediator in short-term memory (STM), and slow on task alternation trials, where it suffers interference from the previous mediator in STM. Thus, the remaining switch costs can be accounted for by priming of bottom-up retrieval processes. Evidence from modeling and simulation data show that switch costs in the explicit task cuing procedure may be accounted for strictly with bottom-up processes (Schneider \& Logan, in press) and thus fail to provide strong evidence for top-down control of performance in the explicit task cuing procedure.

Performance in the voluntary task switching procedure differs from that in the explicit task cuing procedure in a number of ways. Subjects must implement some degree of top-down control in the voluntary task switching procedure, and this fact provides us some insight into the switch costs. The switch costs cannot be the result of bottom-up cue encoding processes because there is no external cue. However, it is possible that other passive mechanisms contribute to the switch costs. The target stimulus may trigger bottom-up episodic retrieval or priming of S-R mappings that produce interference on task alternation trials (Allport \& Wylie, 2000). However, the reduction in switch costs with increasing SOA argues against this account. Alternatively, the switch costs may reflect facilitatory priming of memory based processes involved in performance, such as the mediation process described by Logan and Bundesen (2004). After subjects have chosen the task to perform, they may use a combination of the task name and the target stimulus to retrieve the response from memory. Thus, retrieval of the task name may be a critical aspect of task choice. Retrieval of the task name may be accomplished more quickly if it was already in STM, as on task repetition trials, than if it must be retrieved from long term memory, as on task alternation trials. The reduction in switch costs with increasing SOA would be consistent with this account because the differences in the likelihood that the task name will be retrieved prior to the onset of the target stimulus will decrease as SOA increases (for a detailed model of this SOA effect, see Schneider \& Logan, in press).

Alternatively, voluntary switch costs may reflect active topdown processes. There are two points at which these control processes might occur. They may be involved in preparing for the upcoming task after the task has been chosen; in other words, they may be top-down configuration of task set as proposed in theories that posit top-down accounts of switch costs. Another possibility is that switch costs result from the time required to choose the task to perform on the upcoming trial. This account assumes that the choice process takes some time and that this process does not occur with equal frequency on both task repetition and task alternation trials. Subjects may not choose which task to perform on every trial but rather may only choose on trials on which they switch from the current task. That is, they may perform one task 
for a number of trials until they make the decision to switch to the other task. From this perspective, switch costs could reflect the costs associated with the act of choosing the task on task alternation trials. We address this hypothesis in the following experiments.

\section{Experiment 4}

The cost of a voluntary task switch could reflect the time required to reconfigure the task set on task alternation trials or it could reflect the time to choose to switch tasks. Subjects could repeat tasks by default and make a choice only on trials on which they switch tasks. Experiment 4 was conducted to distinguish between these possibilities. Subjects performed runs of trials in which they chose the task on the first trial in a run and repeated the chosen task on subsequent trials. In some ways, this procedure is similar to alternating runs (Rogers \& Monsell, 1995) and extended runs procedures (Gopher et al., 2000). Trial runs were either pairs or triplets; there was either one or two repetitions of the chosen task after the choice trial. There were three kinds of transitions between trials in each run: (a) chosen alternations, in which subjects choose to change tasks on the choice trial, (b) chosen repetitions, in which subjects choose to repeat tasks on the choice trial, and (c) instructed repetitions, in which subjects must repeat the task they chose on the preceding choice trial. If subjects only actively choose the task on trials in which they switch tasks, then chosen repetitions and instructed repetitions should not differ from each other. However, if subjects actively choose the task on all choice trials, then the contrast between chosen repetitions and instructed repetitions measures the cost of making this choice, and the contrast between chosen alternations and chosen repetitions measures the cost of switching tasks.

\section{Method}

Subjects. Sixteen subjects participated in Experiment 4.

Design. Trials appeared in runs in which the first trial in the run was performed under voluntary task switching instructions, and the subsequent trials were instructed to be repetitions of the chosen task. The runs of trials occurred either as pairs or triplets. For the first trial in each run, trials were assigned to task repetition and task alternation conditions on the basis of the task performed on the previous choice trial. The remaining trials, one trial in the pair condition and two trials in the triplet condition, were instructed repetitions. Thus, for the pairs, there were three transition conditions: chosen alternation, chosen repetition, and instructed repetition; for the triplets, there were four transition conditions: chosen alternation, chosen repetition, instructed repetition-1, and instructed repetition-2. There were four RSIs: 0, 300, 600, and $900 \mathrm{~ms}^{1}{ }^{1}$

Apparatus, stimuli, and procedure. The background was light gray, and the warning box colors were blue and dark gray. The warning box was blue during the first trial in each run, which indicated that the subject was to choose the task, and the warning box was dark gray for the remaining trials, which indicated that the subject was to repeat the chosen task. Unlike the previous experiments, the warning box did not disappear between trials but rather was on the screen continuously to generate continuity between the two or three trials in a run.

Following practice of the subordinate level tasks, subjects received the instructions for the switching procedure. The instructions for the first trial of each run were the voluntary task switching instructions provided in the earlier experiments, and the instructions for the remaining trials in a run were to repeat the chosen task. Subjects completed 12 blocks of 32 runs for both the pair and triplet conditions, the order of which was counterbalanced across subjects.

\section{Results and Discussion}

Choice probability analyses. For the first trial of each run, choice probability analyses were performed. The mean probability of doing the parity task was 0.510 for the pairs and 0.505 for the triplets and included the chance value of 0.5 in the $95 \%$ confidence interval for both conditions. The choice of task as a function of the target stimulus is shown in Table 1. At the group level, there was no significant effect of the target digit on task choice, $F(7,105)=$ $1.3, p>.2, M S E=0.0033$. At the level of individual subjects, 3 of 16 subjects showed a significant relationship between the target digit and the task chosen. These effects showed no consistent pattern across subjects. Because the warning box color was the same for all choice trials, there were no contingency analyses that involved warning box color.

The proportions of task repetitions are shown in Table 2 as a function of RSI, separated by pair and triplet conditions. In the pair condition, the chance probability of a repetition fell below the $95 \%$ confidence interval for the 0-, 300-, and 600-ms RSIs. In the triplet condition, the chance probability of a repetition fell below the $95 \%$ confidence interval for the 0-ms RSI but not for the longer time intervals. The effects of length of run and RSI were examined in a 2 (run length: pairs or triplets) $\times 4$ (RSI: 0, 300, 600, and 900 ms) repeated measures ANOVA. The main effect of RSI was significant, $F(3,45)=19.5, p<.05, M S E=0.0035$, with pairwise comparisons showing that the repetition probability showed no significant difference between the 0- and 300-ms RSI conditions but was significant in all other pairwise comparisons. The main effect of run length was also significant, $F(1,15)=5.9$, $p<.05, M S E=0.0209$. The proportion of repetitions was greater when the trials appeared in pairs, 0.60 , than when they appeared in triplets, 0.54 .

Contingencies between choice behavior and potential response repetitions and choice behavior and target repetitions were also examined. Mean probability values for task repetitions for these contingencies are given in Table 3. Across subjects, there was a significantly higher proportion of task repetitions for potential response repetitions, $F(1,15)=5.2, p<.05, M S E=0.0014$. In the individual analyses, this effect was significant in 1 of 16 subjects. There was no significant effect of target repetition on choice behavior at either the group, $F(1,15)<1$, or individual level.

$R T$ analyses. The mean RTs for each transition condition are plotted as a function of RSI in Figure 4, separated by pairs (A) and triplets (B). For choice trials in both the pair and triplet conditions,

\footnotetext{
${ }^{1}$ In Experiment 4, the RSI was manipulated rather than the SOA between the warning box and target as in the previous experiments, because the warning box was not removed from the screen between trials Thus, the RSI values represent the full time interval between trials, which were $100 \mathrm{~ms}$ shorter than the time intervals between trials in the earlier experiments in which there were both a 100-ms blank interval between trials and a variable SOA with the same time intervals that were used for the RSIs in the current experiment. In Experiment 5, the RSI values were increased by $100 \mathrm{~ms}$ to match the overall time intervals from Experiments 1-3.
} 
A

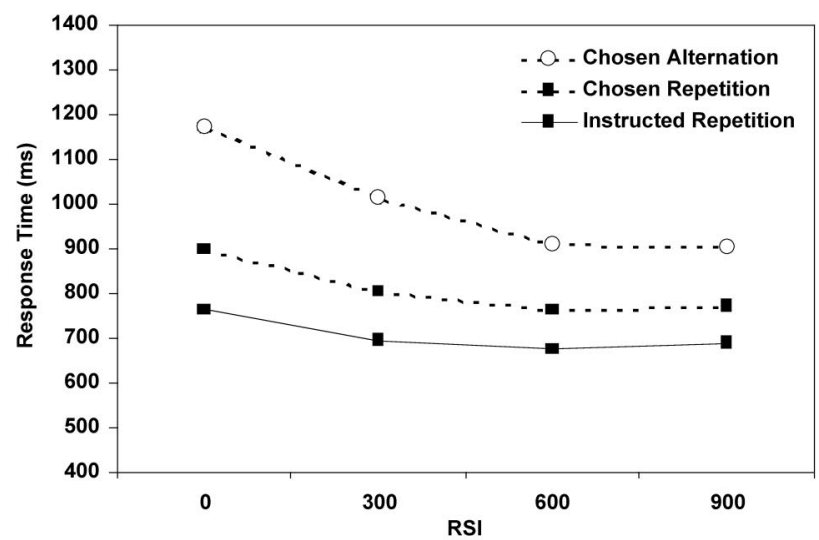

B

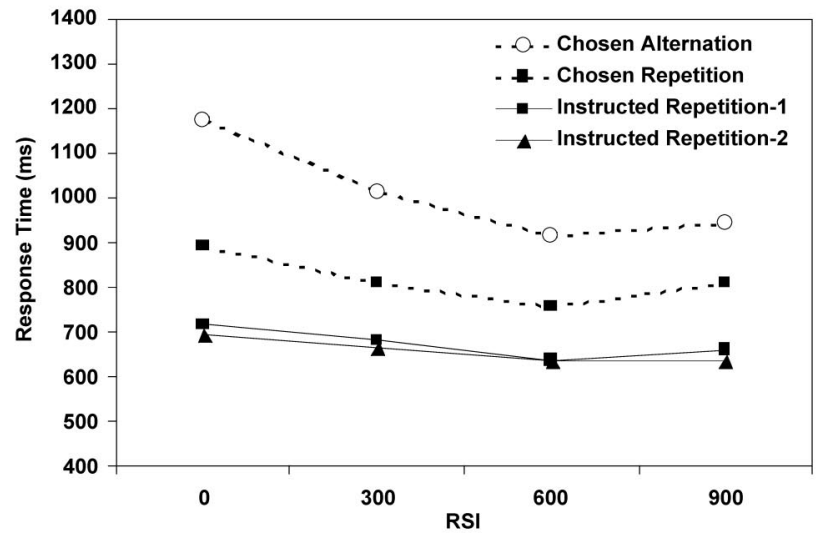

Figure 4. Experiment 4 pairs (A) and Experiment 4 triplets (B): Mean response time in milliseconds as a function of response to stimulus intervals (RSIs) for the instructed repetition (solid lines with solid squares [1] and solid triangles [2]), chosen repetition (dotted lines with solid squares), and chosen alternation (dotted lines with open circles) trials.

the standard switch costs were seen, with subjects responding more quickly when they chose to repeat the task from the previous run than when they chose to switch tasks. In addition to the switch costs, there were also substantial choice costs, with subjects responding more quickly on instructed repetitions than on chosen repetitions.

The RT data were analyzed separately for the two run lengths: Pairs were analyzed in a 3 (task transition: chosen alternation, chosen repetition, and instructed repetition) $\times 4$ (RSI: $0,300,600$, and $900 \mathrm{~ms}$ ) repeated measures ANOVA, and triplets were analyzed in a 4 (task transition: chosen alternation, chosen repetition, instructed repetition-1, and instructed repetition-2) $\times 4$ (RSI: 0 , 300,600 , and $900 \mathrm{~ms}$ ) repeated measures ANOVA. For trials that appeared in pairs, there were significant main effects of task transition, $F(2,30)=46.7, p<.05, M S E=32,647.5$, and RSI, $F(3,45)=45.0, p<.05, M S E=5,809.9$. The interaction of these two factors was significant, $F(6,90)=9.6, p<.05, M S E=$ 3,554.5. This interaction was broken down further to look at the time course of switch costs and choice costs separately. In the comparison of chosen switch and chosen repetition conditions, the planned contrast that tested the linear component of the interaction between task transition and RSI was significant, $F(1,45)=25.0$, $p<.05, M S E=3,554.5$; switch costs decreased monotonically with increasing RSI ( switch costs $=282,214,154$, and $145 \mathrm{~ms}$, for 0-, 300-, 600-, and 900-ms RSIs, respectively). In the comparison of chosen repetition and instructed repetition conditions, the interaction between task transition and RSI was again significant, $F(3$, $45)=4.3, p<.05, M S E=3,554.5$; choice costs decreased monotonically with increasing RSI (choice costs $=140,112,88$, and $83 \mathrm{~ms}$, for 0-, 300-, 600-, and 900-ms RSIs, respectively).

In the triplets, the main effect of task transition was significant, $F(3,45)=42.3, p<.05$, with pairwise comparisons showing significant decreases in RT from chosen alternations $(M=1,015$ $\mathrm{ms})$ to chosen repetitions $(M=817 \mathrm{~ms})$ and from chosen repetitions to instructed repetitions $(M \mathrm{~s}=674$ and $659 \mathrm{~ms}$ for first and second repetitions, respectively, which did not differ from each other). The main effect of RSI was also significant, $F(3,45)=$ 42.7, $p<.05, M S E=4,940.2$, as was the interaction of the two factors, $F(9,135)=8.4, p<.05, M S E=3,297.2$. Again, the interaction was broken down to look at the effect of RSI on switch costs and choice costs. In the planned contrasts of chosen alternation and chosen repetition conditions, the linear trend between task transition and RSI was significant, $F(1,45)=28.7, p<.05$, $M S E=3,297.2$; switch costs decreased monotonically with increasing RSI (switch costs $=284,206,164$, and $136 \mathrm{~ms}$, for 0-, 300-, 600-, and 900-ms RSIs, respectively). Choice costs, measured as the difference between chosen repetition condition and the instructed repetition- 1 and instructed repetition- 2 conditions, did not have a significant linear trend with increasing RSI, $F(3,45)=$ $1.5, p>.05, M S E=3,297.2$; the decrease in choice costs was not monotonic, increasing at the longest RSI (choice costs $=186,139$, 118, and $161 \mathrm{~ms}$, for 0-, 300-, 600-, and 900-ms RSIs, respectively).

For both pairs and triplets, there were significant switch costs when comparing chosen repetitions and chosen alternations. These switch costs were similar in magnitude and time course to the switch costs seen in the voluntary task switching conditions in Experiments 1-3. There were also significant differences between chosen repetitions and instructed repetitions. These differences reflected costs associated with the voluntary act of choosing the task to perform. Additionally in the triplet condition, there was no significant difference between first and second instructed repetitions. Thus, the position in the run of trials did not affect performance beyond the difference between chosen repetitions and instructed repetitions. This limitation of choice costs to the first trial in the run is in line with previous work that has shown that costs are limited to the first trial in a run of predictable task switches, as in this experiment (Monsell, Sumner, \& Waters, 2003).

The choice probability analyses shed some light on how the voluntary choice was made. Subjects were more likely to repeat the task on choice trials if the trials appeared in pairs than if they appeared in triplets. This result suggests that subjects may make the choice to repeat or switch tasks with an algorithm that involves counting the number of times a task was performed, regardless of whether the performance was chosen or instructed. Subjects may hold in memory some number of previous trials and use this information to determine which task to choose (Rapoport \& Budescu, 1997). Longer runs of instructed repetitions decrease the number of choice trials and increase the proportion of task repetitions within the previous $n$ trials that can be retained in working 
memory. Either or both of these effects could shift the repetition bias in the direction seen in this experiment.

\section{Experiment 5}

In Experiment 4, chosen repetitions were substantially slower than instructed repetitions. We interpret the RT difference as reflecting the time required to make a voluntary choice about which task to perform. However, the chosen and instructed repetition conditions also differed in terms of the color of the warning box. Subjects were told to choose the task when the warning box was blue and repeat the task when the warning box was gray. Although the pairs and triplets were run in separate blocks so that subjects knew the run length and could anticipate choice and instructed repetition trials, they may have used the blue warning box as an external cue indicating that a choice should be made. Indeed, the color of the warning box was intended to help subjects keep track of whether the trial required a choice or a repetition. However, subjects may have adopted a strategy by which they performed task repetitions unless they saw a blue box, in which case they chose the task. This approach would introduce a difference between voluntary and instructed repetitions in terms of cue encoding. We know from previous research that cue encoding can slow performance at the start of a run of trials even when a task is repeated from one run to the next (Altmann, 2002; Arrington \& Logan, 2004b; Gopher et al., 2000; Logan \& Bundesen, 2003, 2004). We address this issue in Experiment 5.

Subjects performed pairs of trials in which they chose the task to perform on the first trial in the pair and then repeated the task on the second trial, as in the pair condition in Experiment 4. However, in this experiment there was no external cue that indicated whether a target was the first or second in the pair. A gray warning box appeared on the screen at the start of a block of trials and remained on the screen throughout the block without changing color. If the RT difference between chosen and instructed repetitions in Experiment 4 reflected cue encoding on choice trials, then the difference should disappear in the current experiment; however, if the RT difference reflected the time required to make the voluntary choice, then the difference should remain in the current experiment.

\section{Method}

Subjects. Seventeen subjects participated in Experiment 5. Data from 1 subject were excluded from analyses because of the subject's failure to understand the random generation instructions.

Design. Trials appeared in pairs in which the first trial in the pair was performed under voluntary task switching procedures, and the second trial was an instructed repetition of the chosen task. The first trial in each pair was assigned to chosen repetition and chosen alternation conditions on the basis of the task performed on the current trial and the previous choice trial. Thus, there were three transition conditions: chosen repetition, chosen alternation, and instructed repetition. There were four RSIs: 100, 400, 700, and $1,000 \mathrm{~ms}$.

Apparatus, stimuli, and procedure. The apparatus and stimuli were the same as in Experiment 4, except as noted. The warning box color was dark gray. The warning box remained on the screen throughout the block to eliminate any possible cue to the subject that would distinguish choice trials from instructed repetitions.

Following practice of the subordinate level tasks, subjects received the instructions for the switching procedure. The instructions for the first trial of each pair were the voluntary task switching instructions. Subjects were told that after they chose a task they should perform the task on a pair of trials and then choose again at the start of the next pair of trials. Because there was no external cue reminding subjects of which trial in a pair they were performing (i.e., choice or instructed repetition trial), they received extra instructions that stressed the importance of maintaining the pair structure in memory during the block of trials. In addition, the number of trials per block was reduced to limit the chance of subjects forgetting where they were in the pairs and thus choosing a task when they should be performing an instructed repetition. ${ }^{2}$ Subjects completed 64 blocks with 20 trials, or 10 pairs, per block.

\section{Results and Discussion}

Choice probability analyses. For the first trial of each pair, choice probability analyses were performed. The mean probability of doing the parity task was 0.509 and excluded the chance value of 0.5 from the $95 \%$ confidence interval. The choice of task as a function of the target stimulus is shown in Table 1. There was no significant effect of the target digit on task choice, $F(7,105)=1.1$, $p>.3, M S E=0.0038$. At the level of individual subjects, 1 of 16 subjects showed a significant relationship between the target digit and the task chosen. Because the warning box was the same for all choice trials, there were no contingency analyses that involved warning box color.

The proportions of task repetitions as a function of RSI are shown in Table 2 . The chance probability of a repetition fell below the $95 \%$ confidence interval for the 100- and 400-ms RSI but not for the longer time intervals. The effect of RSI was significant, $F(3,45)=7.7, p<.05, M S E=0.0021$. Contingencies between choice behavior and target repetition and potential response repetition were also examined. Mean probabilities for task repetitions for these contingencies are given in Table 3. Across subjects, there was no significant difference in repetition probability based on potential response repetition, $F(1,15)=1.9, p=.19, M S E=$ 0.0008 . In the individual analyses, 1 of 16 subjects was significantly more likely to repeat the task if a response repetition would occur. Across subjects, there was a tendency toward a higher repetition probability if the target repeated, $F(1,15)=3.5, p=$ $.08, M S E=0.0039$. In the individual analyses, the effect was significant for 4 of 16 subjects.

$R T$ analyses. The mean RTs for each transition condition are plotted as a function of RSI in Figure 5. The pattern of results was similar to that seen in Experiment 4, showing both switch costs and choice costs. The RT data were analyzed in a 3 (task transition: chosen alternation, chosen repetition, and instructed repetition) $\times$ 4 (RSI: 100, 400, 700, and 1,000 ms) repeated measures ANOVA. Both main effects were significant: task transition, $F(2,30)=$ 89.3, $p<.05, M S E=21,754.0$, and RSI, $F(3,45)=27.9, p<.05$, $M S E=4,979.7$. The interaction of the two variables was significant, $F(6,90)=5.9, p<.05, M S E=2,598.6$. This interaction was broken down to examine the time course of the switch costs

\footnotetext{
${ }^{2}$ To address the concern that subjects might "get off sequence" of choice and instructed repetition trials, we examined the data in several ways: (a) removing only error trials as in the other experiments, (b) removing all trials in a block following an error, and (c) removing all blocks that contained an error. In all cases, the pattern of data and significance of statistical tests remained the same. The data presented are those with only error trials removed as in the other experiments in this article.
} 


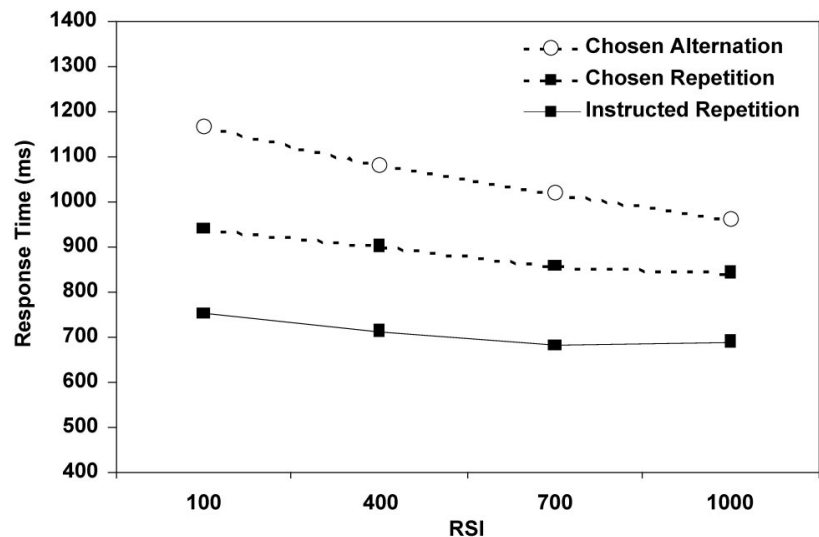

Figure 5. Experiment 5: Mean response time in milliseconds as a function of response to stimulus intervals (RSIs) for the instructed repetition (solid lines with solid squares), chosen repetition (dotted lines with solid squares), and chosen alternation (dotted lines with open circles) trials.

and choice costs separately. In the comparison of chosen alternation and chosen repetition conditions, the planned contrast that tested the linear component of the interaction between task transition and RSI was significant, $F(1,45)=17.2, p<.05, M S E=$ 2,598.6; switch costs decreased monotonically with increasing RSI (switch costs $=226,179,163$, and $120 \mathrm{~ms}$, for 100-, 400-, 700-, and 1,000-ms RSIs, respectively). In the comparison of chosen repetitions and instructed repetitions, the planned contrast that tested the linear component of the interaction between task transition and RSI was not significant, $F(1,45)=2.3, p=.14, M S E=$ 2,598.6, although the effect of RSI on choice costs was monotonic (choice costs $=189,189,175$, and $153 \mathrm{~ms}$, for 100-, 400-, 700-, and 1,000-ms RSIs, respectively).

The switch costs and choice costs in Experiment 5 were similar in pattern to the costs in Experiment 4. There was an overall shift in RT; subjects were slower to respond in Experiment 5 for all three task transitions. This shift may be due to the extra requirement of keeping track of the pairs of trials, because there was no external cue that distinguished the choice and instructed repetition trials. The need to hold the pair structure in working memory and continually update the current position in a pair may have slowed performance overall. Also, the blocks of trials were substantially shorter in Experiment 5 than in the Experiment 4 (20 trials vs. 64 or 96 trials, respectively). If a general speed up occurs over trials in a block, then the shorter blocks could have caused the slower RTs. However, even with the overall shift in RTs, switch and choice costs were about the same as in Experiment 4. Thus, this experiment rules out the cue-encoding-cost interpretation of the choice costs in Experiment 4. In the current experiment with no cue, the difference between chosen and instructed repetitions remained.

The comparison between the chosen and instructed repetitions captures the time necessary for carrying out a voluntary act of choosing a task. We would expect that this top-down process may be carried out at least in part during the RSI, such that choice costs decrease with increasing RSI. The effect of RSI on choice cost generally showed this pattern. In Experiment 4, the interactions between choice costs and RSI had a significant linear trend for the pairs but not for the triplets. For the pairs, there was a monotonic decrease in choice costs with increasing RSI, but for the triplets, choice costs decreased over the first three RSIs and then increased at the 900-ms RSI. This increase at the longest RSI may be the result of a general slowing in all conditions at the longest RSI because of foreperiod effects (Luce, 1986). In Experiment 5, there was again a slight decrease in choice costs with increasing RSI, but this effect was not significant.

The choice costs measured in Experiments 4 and 5 provide some insight into the nature of the switch costs in the voluntary task switching procedure. They rule out the hypothesis that subjects make a voluntary choice only on switch trials and then perform the same task by default, without voluntary choice, on subsequent repetition trials. If this was the case, then chosen repetitions and instructed repetitions should have been equivalent, and they were not. Consequently, the switch costs cannot be attributed to choices made on switch trials that are not made on repetition trials. However, chosen repetitions may reflect a mixture of trial types, ones on which subjects chose to repeat the task from the previous trial and ones on which subjects failed to choose and repeated the task by default. If such a mixture occurred, then the difference between task repetitions and task alternations might reflect faster RTs on the proportion of chosen repetitions in which the task was repeated without a choice. However, this mixture model predicts that the standard deviations should be significantly larger for chosen repetitions than for the other conditions (Townsend \& Ashby, 1983, p. 264), which was not the case in any of the current data sets.

Another possibility is that the choice process is faster for chosen repetitions than for chosen alternations. Choosing to perform a task may involve retrieval of the task name (i.e., "Even-Odd" or "Low-High"). On task repetitions, the choice is faster because the task name is already in STM, and retrieval is facilitated. On task alternations, the choice is slower because retrieval is not primed or it suffers interference from the task name in STM, or both. Switch costs may be affected by such differences in the time to retrieve the task name. We examine this possibility in Experiment 6.

\section{Experiment 6}

In Experiment 6, we modified the voluntary task switching procedure to allow us to directly assess the choice process on task repetitions and task alternations. Each trial involved two responses: a choice response and a task response. The trial began with a prompt. Subjects responded to the prompt with a key press that indicated which task they were going to perform on the upcoming target. Following the choice response, the target appeared, and subjects made the second key press, performing the chosen task on the target digit. Thus, this procedure allowed us to examine the choice process separately from task performance and to consider whether the choice processes differed on task repetitions and task alternations. If choice processes are slower on switch trials than on repetition trials, then the switch costs seen in the voluntary task switching procedure may result from choice processes.

\section{Method}

Subjects. Nineteen subjects participated in Experiment 6. Data were excluded from three subjects: One subject failed to produce an adequate 
number of task alternations (proportion of task repetitions for this subject was 0.85 ); one subject had accuracy below $90 \%$; and one subject had an excessively large number of long RTs.

Design. Trials were sorted into task repetition and task alternation conditions on the basis of the task indicated by the choice response on trial $n$ and trial $n-1$. There were two different RSI manipulations: the time from the task response on trial $n-1$ to the onset of the prompt on trial $n$, and the time from the choice response to the onset of the target within trial $n$. Values for both time intervals were randomly selected from 100-, 400-, 700-, and 1,000-ms RSIs.

Apparatus, stimuli, and procedure. The prompt and target stimuli appeared in black on a light gray background. The prompt was a "?" presented in the same size and font as the target digits that were identical to the previous experiments. No warning boxes occurred in this procedure. Responses were made with the index and middle fingers of each hand on the $d, f, j$, and $k$ keys. The choice response was made with one hand and the task response with the other. Therefore, unlike in previous experiments in which the responses for the two subordinate level tasks were univalent (i.e., mapped to unique key presses), the task responses in the current procedure were bivalent. The mapping of type of response to hands and specific responses to fingers was counterbalanced across subjects such that all combinations of responses were used.

After practice of the subordinate level tasks, subjects received the voluntary switching instructions. Subjects were instructed to first indicate the task they had chosen to perform in response to the prompt and then to perform that task on the subsequent target digit. Other than the key press response required for the choice of task, the voluntary task switch instructions were the same as those used in previous experiments. The subjects were instructed to respond as quickly and accurately as possible on both the choice and task responses. Following a brief block of practice trials, subjects completed 12 blocks of 64 trials.

\section{Results and Discussion}

Choice probability analyses. The mean probability of doing the parity task was 0.505 and included the chance value of 0.5 from the $95 \%$ confidence interval. Because the prompt was the same on all trials and the target did not appear until after the choice of task was made, there were no external stimuli that could have affected task choice or repetition probability. The only variable that could have affected task choice was the RSI. The proportions of task repetitions as a function of RSI are shown in Table 2. The chance probability of a repetition fell below the $95 \%$ confidence interval for the 100- and 400-ms RSI but not for the longer time intervals. The effect of RSI was significant, $F(3,45)=3.4, p<$ $.05, M S E=0.001$.

$R T$ analyses. The mean RTs for choice and task responses are plotted as a function of RSI in Figure 6. The effects of task transition and RSI differed substantially for the two types of responses. For the choice response, the RTs for task repetition and task alternation conditions were virtually overlapping, except at the shortest RSI, where the choice on task alternations took $52 \mathrm{~ms}$ longer than the choice on task repetitions. For both task repetitions and task alternations, the time-course functions showed a substantial decrease in choice RT as RSI increased from $100 \mathrm{~ms}$. For the task response, the difference in RTs between the task repetitions and task alternations showed large switch costs that decreased only slightly with increasing RSI.

The data were analyzed for the choice and task responses separately in two 2 (task transition: repetition and alternation) $\times 4$ (RSI: 100, 400, 700, and 1,000 ms) repeated measures ANOVAs. For the choice RTs, the main effect of RSI was significant, $F(3$,

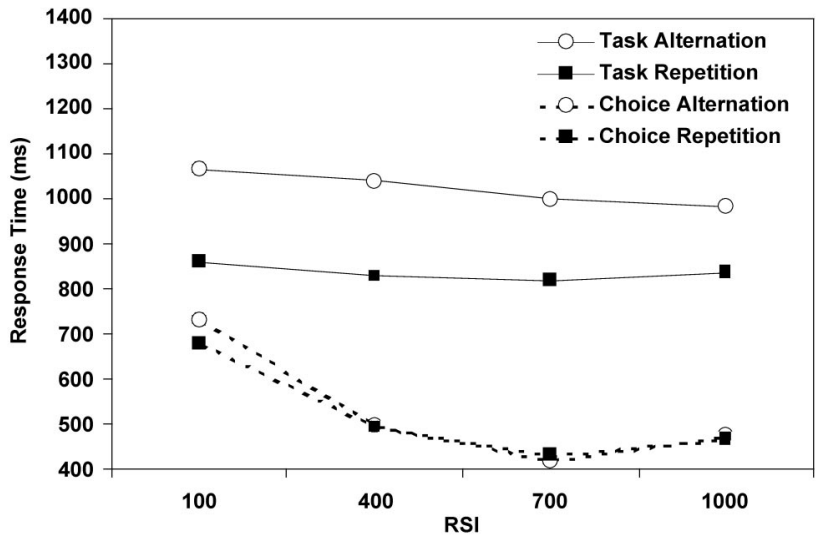

Figure 6. Experiment 6: Mean response time in milliseconds as a function of response to stimulus intervals (RSIs) for the choice response repetition (dotted lines with solid squares), choice response alternation (dotted lines with open circles), task response repetition (solid lines with solid squares), and task response alternation (solid lines with open circles) trials.

$45)=34.3, p<.05, M S E=14,409.1(M \mathrm{~s}=706,495,426$, and $470 \mathrm{~ms}$, for 100-, 400-, 700-, and 1,000-ms RSIs, respectively). Overall, the comparison of task transition conditions showed no significant switch costs, $F(1,15)=2.2, p<.05, M S E=2,164.7$, but task transition interacted significantly with $\operatorname{RSI}, F(3,45)=$ 4.8, $p<.05, M S E=1,309.9$. Pairwise comparisons showed that the switch costs were significant only at the 100-ms RSI (switch costs $=52,1,-13$, and $7 \mathrm{~ms}$, for the 100-, 400-, 700-, and 1,000-ms RSIs, respectively).

For the task RTs, the robust switch costs were reflected in a highly significant main effect of task transition, $F(1,15)=61.9$, $p<.05, M S E=17,881.1(M \mathrm{~s}=836$ and $1,021 \mathrm{~ms}$ for task repetitions and task alternations, respectively). The main effect of RSI was also significant, $F(3,45)=4.0, p<.05, M S E=5,108.3$ $(M \mathrm{~s}=963,932,909$, and $910 \mathrm{~ms}$, for the 100-, 400-, 700-, and 1,000-ms RSIs, respectively). The interaction between the two factors was marginally significant, $F(3,45)=2.7, p=.06$, $M S E=2,709.4$, with switch costs generally decreasing with increasing RSI (switch costs $=208,211,179$, and $146 \mathrm{~ms}$, for the 100-, 400-, 700-, and 1,000-ms RSIs, respectively). The planned contrast examining the linear trend in this interaction was significant, $F(1,45)=6.9, p<.05, M S E=2,709.4$.

The strikingly different effects of task transition and RSI on choice and task RTs demonstrate that these responses capture different aspects of the control processes involved in the voluntary task switching procedure. On the basis of the results from Experiments 4 and 5, it was possible that the difference between chosen repetitions and chosen alternations resulted from differences in choice processes, with subjects requiring more time to choose to perform a task that was not performed on the previous trial. The current experiment rules out that explanation of the switch costs. Choice RTs for task repetitions and task alternations did not differ except at the shortest RSI, and this difference was not large enough to contribute substantially to the switch costs measured in other experiments. However, there remained large switch costs for the task RT measure. 


\section{General Discussion}

Task switching methodologies have become widely used in the study of executive control. However, there has been substantial controversy about whether switch costs result from top-down processes involved in active reconfiguration of the cognitive system or from bottom-up processes that result in interference or facilitation from recently activated task sets. In the current research, we addressed this controversy from a new angle using a voluntary task switching procedure. This procedure differs from other task switching procedures in that it requires that subjects choose the task to perform on every trial. In this series of experiments, we addressed two questions. First, do subjects emit choices or allow them to be elicited by stimuli in the environment? Across all experiments, subjects' choice behavior was largely uninfluenced by external stimulus events, suggesting that task choice is controlled in a top-down fashion. These results support the assumption that the voluntary task switching procedure ensures a top-down component of task performance by requiring the subject to make this choice. Second, do such top-down acts of control produce switch costs? The simple answer to this question is clearly "yes." Switch costs were observed consistently across all of the experiments, which led to the further question: What is the cause of voluntary switch costs? To address this question, we compared the switch costs in voluntary task switching and explicit task cuing paradigms in Experiments 1-3, and we separated task choice costs from task switch costs in Experiments 4-6.

\section{Task Choice as a Top-Down Process}

The analyses of the contingencies between choice behavior and external stimuli were critical for determining the nature of the choice process. There were two external stimuli that might influence choice: the warning box and the target digit. The warning box color had very small and inconsistent effects on task choice, even when tasks and colors had previously been associated during the explicit task cuing condition, and thus might have elicited a particular response based on episodic retrieval of associations between warning box color, target stimulus, and response (Waszak et al., 2003; Wylie \& Allport, 2000). Likewise, the effect of the target stimulus was also small and inconsistent. The effect of target identity on task choice was significant for only two of the five experiments, as was the tendency to repeat a task more frequently if the target digit repeated. Overall, these analyses suggest that choice behavior was largely uninfluenced by stimulus-driven processes and support the assumption that subjects made voluntary choices about the task to perform and whether to repeat or switch tasks in a top-down fashion.

The one factor that did have a significant and systematic effect on repetition probabilities was the time interval between trials. Repetition bias decreased as the time between trials increased in every experiment. This shift with RSI suggests possible explanations for the repetition bias. The act of choosing which task to perform requires time, as was demonstrated in comparing chosen and instructed repetitions in Experiments 4 and 5 and in the choice RTs in Experiment 6. If a target appears very quickly after the response on trial $n-1$, then subjects may perform the currently prepared task without making a voluntary choice. Task repetitions may be the default behavior. Subjects may fail to complete a choice about the task to perform on shorter RSIs and thus perform the default behavior more frequently, leading to a higher repetition probability at shorter RSIs. However, the repetition bias seen in Experiment 6 argues against this hypothesis, because subjects were responding to the prompt, which was the same on every trial, rather than to the target stimulus.

We suggest that the choice probability reflects a competition between a representativeness heuristic (Rapoport \& Budescu, 1997) and an availability heuristic (Baddeley, 1996). The representativeness heuristic operates by evaluating the $n$ previous trials held in STM and choosing the element that makes the series most representative of the subjects' idea of a random sequence. Rapoport and Budescu (1997) developed a model based on this idea that adequately accounts for the alternation bias commonly found in random generation experiments. The results from Experiment 4 show support for such a comparison from STM to a representation of randomness: The repetition bias decreased when subjects performed triplets of the chosen task, which increased the proportion of repetitions in the $n$ trials maintained in STM.

Baddeley and colleagues have argued that random sequence generation involves executive control processes (Baddeley, 1996; Baddeley, Chincotta, \& Adlam, 2001; Baddeley, Emslie, Kolodny, \& Duncan, 1998) that must overcome habitual or more easily retrieved responses. They used random sequence generation as a secondary task to track the executive control demands of a primary task. According to their logic, tasks that require more executive control lead to less random behavior in the random sequence generation task. In the voluntary task switching procedure, the random production of a sequence of tasks was intermixed with the performance of the subordinate level tasks and, more importantly, with the executive control processes involved in configuring the cognitive system to perform the chosen task. From the perspective of Baddeley and his colleagues, the repetition bias may be seen as evidence of competition between two executive control processes-randomly choosing the task and switching between tasks-which is more severe at short RSIs. Thus, the representativeness and availability heuristics work in opposition with each other, with the availability heuristic dominating at the shorter RSIs.

The choice behavior analyses strongly support the assumption that task choice is a top-down process. The RT data address the question of whether voluntary choice produces switch costs and what the nature of those costs might be. Across all six experiments, subjects showed substantial switch costs in the voluntary task switching procedure. The time-course functions of the switch costs were consistent across the experiments, showing monotonic decreases in switch costs as the interval between trials increased. This decrease over time is characteristic of a timeconsuming, set-switching process that is carried out at least in part during the interval between trials (Logan \& Bundesen, 2003; Meiran, 1996), which is consistent with top-down theories of task switching (Logan \& Gordon, 2001; Rogers \& Monsell, 1995). Subjects also showed substantial choice costs calculated from the difference between chosen repetitions and instructed repetitions in Experiments 4 and 5 and from the choice RTs in Experiment 6. We interpret these RT data as evidence that topdown control results in switch costs. The implications of this conclusion for the debate between top-down and bottom-up theories of task switching requires some consideration of the demands 
that the voluntary task switching procedure places on the top-down control of behavior.

\section{Environmental Support and Task Switching}

Task switching paradigms differ in the amount of environmental support provided by the procedure. Environmental support refers to the extent to which the environment provides the information necessary for an appropriate response so that cues in the environment can elicit the response directly (Craik, 1983; Einstein, McDaniel, Richardson, Guynn, \& Cunfer, 1995). We suggest the following hypothesis: Task switching procedures that involve a high degree of environmental support require little top-down control; procedures that involve a low degree of environmental support require more top-down control.

The explicit task cuing procedure provides the greatest amount of environmental support and the voluntary task switching procedure involves the least. In the explicit task cuing procedure, the combination of the cue and target uniquely specifies a response on each trial: No top-down control is necessary for switching tasks in this procedure (Logan \& Bundesen, 2003). In the voluntary task switching procedure, the targets are the only external stimuli presented during the experiment, and they are ambiguous. Topdown control is required to determine which task to perform. Other procedures are intermediate. The extended runs procedure provides some environmental support. It presents a cue at the beginning of each run, which must then be maintained in memory throughout the run. This procedure provides less environmental support than the explicit task cuing procedure because it imposes a need to retrieve the task on task repetition trials (Altmann \& Gray, 2002; Gopher et al., 2000). The alternating lists and alternating runs procedures involve less environmental support. They require subjects to switch between tasks in a regular sequence, which involves holding in memory the current trial and several previous trials, depending on run length, to switch tasks appropriately (Jersild, 1927; Rogers \& Monsell, 1995). Note that when the alternating lists or alternating runs procedures include an external cue that serves as a reminder of the task sequence, such as the location of the target, these procedures become variations of the explicit task cuing procedure in which there is complete environmental support. The task span procedure involves even less environmental support than the alternating runs and alternating tasks procedures (Logan, 2004). It requires subjects to commit a sequence of tasks to memory and then produce that sequence on a series of target stimuli. As the environmental support for each task decreases, the need for top-down control increases. Thus, when chasing the elusive homunculus it is critical to use a procedure with little environmental support so that task performance requires top-down control.

\section{Top-Down and Bottom-Up Accounts of Switch Costs}

The voluntary task switching procedure inverts the usual question in interpreting switch costs, asking "does an endogenous act of control produce switch costs?" instead of "do switch costs reflect an endogenous act of control?" The evidence that voluntary task switching produces strong switch costs shifts the balance of evidence toward top-down interpretations of switch costs and away from bottom-up explanations. Voluntary switch costs decreased substantially as preparation interval increased, confirming the prediction of top-down theories that reconfiguration can occur in the preparation interval before the target appears (Meiran, 1996; Rogers \& Monsell, 1995). This suggests that other procedures that show a reduction in switch costs with increasing preparation interval might also reflect an endogenous act of control, provided that two other conditions are fulfilled. First, the procedure must not provide complete environmental support so that top-down processing is necessary. The explicit task cuing procedure fails to meet this criterion because it provides complete environmental support, and the reduction in switch costs with preparation interval can be explained as a cue encoding benefit (Arrington \& Logan, 2004b; Logan \& Bundesen, 2003, 2004). Second, the reduction in switch costs must not be confounded with the time since the last task was performed. Allport et al. (1994) argued that previous task sets may dissipate over time, mimicking the effects of endogenous processes that occur during the preparation interval. Several experiments have shown reductions in switch costs that are independent of the time since the last task was performed, fulfilling this second condition (Arrington \& Logan, 2004b; Logan \& Bundesen, 2003; Mayr \& Kliegl, 2003; Meiran, 1996; Meiran, Chorev, \& Sapir, 2000).

It is possible that the switch costs we observed in the voluntary task switching procedure are instigated by bottom-up processes, such as proactive interference from previous task sets (Allport et al., 1994) and stimulus-specific priming of previous task sets and responses (Allport \& Wylie, 2000; Waszak et al., 2003; Wylie \& Allport, 2000). However, two considerations mitigate the conclusion that voluntary switch costs are entirely due to bottom-up processes. First, as we just mentioned, voluntary switch costs decrease with increasing preparation interval. Stimulus-specific priming cannot decrease with increasing preparation interval because it is instigated by the target, which appears at the end of the preparation interval. In theory, it represents residual switch costs, which are switch costs that remain at the longest preparation interval after preparation is complete. Some component of voluntary switch costs may represent residual switch costs, but the part that decreases as preparation interval increases cannot be due to the bottom-up processes that produce residual switch costs.

The second mitigating factor stems from a limitation in the evidence for bottom-up switch costs: Bottom-up processes may instigate switch costs, but they do not necessarily resolve them. Bottom-up processes may produce proactive interference and negative priming from previous task sets, but that does not imply that bottom-up processes are responsible for resolving the interference and overcoming the priming. Mayr and Keele (2000) suggested that top-down processes actively inhibit previous task sets. Gilbert and Shallice (2002) and Yeung and Monsell (2003b) proposed computational accounts of proactive interference and negative priming, and these models assume that a top-down control signal is necessary to overcome interference and priming. The control signal is required on both task repetition and task alternation trials, but it takes longer to resolve the competition on alternation trials. This suggests a differential involvement of top-down processes on repetition and alternation trials, as top-down theories predict.

On the balance, then, the evidence suggests that switch costs observed in procedures that show a reduction in switch costs with increasing preparation interval without providing a lot of environmental support may reflect top-down control. This conclusion and 
the evidence of top-down switch costs in the voluntary task switching procedure support top-down theories of task switching, though they do not rule out bottom-up accounts. Researchers studying executive control have tended to make general statements about the underlying causes of switch costs across various task switching procedures. Our view is that the specific experimental parameters in which a task switch takes place is a crucial consideration when accounting for switch costs.

\section{Conclusion}

In the last 10 years, task switching methodologies have become a standard tool in the experimental investigation of the homunculus (Monsell \& Driver, 2000). The voluntary task switching procedure represents an important development of the task switching paradigm. Placing the decision about which task to perform under the control of the subject introduces a new element of top-down control into task performance and, thus, widens the range of control processes that can be studied in task switching procedures. Choice behavior and choice costs, as well as switch costs, become measures that capture the clever homunculus.

\section{References}

Allport, A., Styles, E. A., \& Hsieh, S. (1994). Shifting intentional set: Exploring the dynamic control of tasks. In C. Umilta \& M. Moscovitch (Eds.), Attention \& performance XV: Conscious and nonconscious information processing (pp. 421-452). Cambridge, MA: MIT Press.

Allport, A., \& Wylie, G. (2000). Task switching, stimulus-response bindings, and negative priming. In S. Monsell \& J. Driver (Eds.), Attention \& performance XVIII: Control of cognitive processes (pp. 35-70). Cambridge, MA: MIT Press.

Altmann, E. M. (2002). Functional decay of memory for tasks. Psychological Research, 66, 287-297.

Altmann, E. M., \& Gray, W. D. (2002). Forgetting to remember: The functional relationship of decay and interference. Psychological Science, $13,27-33$.

Arrington, C. M. (2002). Explorations in task space: Similarity effects on task switching. Unpublished doctoral dissertation, Michigan State University, East Lansing.

Arrington, C. M., Altmann, E. M., \& Carr, T. H. (2003). Tasks of a feather flock together: Similarity effects in task switching. Memory \& Cognition, 31, 781-789.

Arrington, C. M., \& Logan, G. D. (2004a). The cost of a voluntary task switch. Psychological Science, 15, 610-615.

Arrington, C. M., \& Logan, G. D. (2004b). Episodic and semantic components of the compound-stimulus strategy in the explicit task-cuing procedure. Memory \& Cognition, 32, 965-976.

Baddeley, A. (1996). Exploring the central executive. Quarterly Journal of Experimental Psychology: Human Experimental Psychology, 49(A), $5-28$.

Baddeley, A., Chincotta, D., \& Adlam, A. (2001). Working memory and the control of action: Evidence from task switching. Journal of Experimental Psychology: General, 130, 641-657.

Baddeley, A., Emslie, H., Kolodny, J., \& Duncan, J. (1998). Random generation and the executive control of working memory. Quarterly Journal of Experimental Psychology: Human Experimental Psychology, 51(A), 819-854.

Craik, F. I. M. (1983). On the transfer of information from temporary to permanent memory. Philosophical Transactions of the Royal Society of London, Series B, 302, 341-359.

Dreisbach, G., Haider, H., \& Kluwe, R. H. (2002). Preparatory processes in the task-switching paradigm: Evidence from the use of probability cues. Journal of Experimental Psychology: Learning, Memory, and Cognition, 28, 468-483.

Einstein, G. O., McDaniel, M. A., Richardson, S. L., Guynn, M. J., \& Cunfer, A. R. (1995). Aging and prospective memory: Examining the influences of self-initiated retrieval processes. Journal of Experimental Psychology: Learning, Memory, and Cognition, 21, 996-1007.

Emerson, M. J., \& Miyake, A. (2003). The role of inner speech in task switching: A dual-task investigation. Journal of Memory and Language, $48,148-168$

Gilbert, S. J., \& Shallice, T. (2002). Task switching: A PDP model. Cognitive Psychology, 44, 297-337.

Gopher, D., Armony, L., \& Greenshpan, Y. (2000). Switching tasks and attention policies. Journal of Experimental Psychology: General, 129, $308-339$.

Hübner, R., Futterer, T., \& Steinhauser, M. (2001). On attentional control as a source of residual shift costs: Evidence from two-component task shifts. Journal of Experimental Psychology: Learning, Memory, and Cognition, 27, 640-653.

Jersild, A. T. (1927). Mental set and shift. Archives of Psychology (Whole No. 89).

Kleinsorge, T., \& Heuer, H. (1999). Hierarchical switching in a multidimensional task space. Psychological Research, 62, 300-312.

Logan, G. D. (2003). Executive control of thought and action: In search of the wild homunculus. Current Directions in Psychological Science, 12, $45-48$.

Logan, G. D. (2004). Working memory, task switching, and executive control in the task span procedure. Journal of Experimental Psychology: General, 133, 218-236.

Logan, G. D., \& Bundesen, C. (2003). Clever homunculus: Is there an endogenous act of control in the explicit task cuing procedure? Journal of Experimental Psychology: Human Perception and Performance, 29, $575-599$.

Logan, G. D., \& Bundesen, C. (2004). Very clever homunculus: Compound stimulus strategies for the explicit task-cuing procedure. Psychonomic Bulletin \& Review, 11, 832-840.

Logan, G. D., \& Gordon, R. D. (2001). Executive control of visual attention in dual-task situations. Psychological Review, 108, 393-434.

Luce, R. D. (1986). Response times. New York: Oxford University Press.

Mayr, U. (2002). Inhibition of action rules. Psychonomic Bulletin \& Review, 9, 93-99.

Mayr, U., \& Keele, S. W. (2000). Changing internal constraints on action: The role of backward inhibition. Journal of Experimental Psychology: General, 129, 4-26.

Mayr, U., \& Kliegl, R. (2000). Task-set switching and long-term memory retrieval. Journal of Experimental Psychology: Learning, Memory, and Cognition, 26, 1124-1140.

Mayr, U., \& Kliegl, R. (2003). Differential effects of cue changes and task changes on task-set selection costs. Journal of Experimental Psychology: Learning, Memory, and Cognition, 29, 362-372.

Meiran, N. (1996). Reconfiguration of processing mode prior to task performance. Journal of Experimental Psychology: Learning, Memory, and Cognition, 22, 1423-1442.

Meiran, N. (2000). Modeling cognitive control in task-switching. Psychological Research, 63, 234-249.

Meiran, N., Chorev, Z., \& Sapir, A. (2000). Component processes in task switching. Cognitive Psychology, 41, 211-253.

Miller, J. (1982). Discrete versus continuous stage models of human information processing: In search of partial output. Journal of Experimental Psychology: Human Perception and Performance, 2, 273-296.

Monsell, S. (2003). Task switching. TRENDS in Cognitive Sciences, 7 , $134-140$.

Monsell, S., \& Driver, J. (2000). Attention \& performance XVIII: Control of cognitive processes. Cambridge, MA: MIT Press.

Monsell, S., Sumner, P., \& Waters, H. (2003). Task-set reconfiguration 
with predictable and unpredictable task switches. Memory \& Cognition, $31,327-342$.

Nickerson, R. S. (2002). The production and perception of randomness. Psychological Review, 109, 330-357.

Psychology Software Tools. (2000). E-prime (Version 1.0). Pittsburgh, PA: Author.

Rapoport, A., \& Budescu, D. V. (1997). Randomization in individual choice behavior. Psychological Review, 104, 603-617.

Rogers, R. D., \& Monsell, S. (1995). Costs of a predictable switch between simple cognitive tasks. Journal of Experimental Psychology: General, 124, 207-231.

Rosenbaum, D. A., Kenny, S. B., \& Derr, M. A. (1983). Hierarchical control of rapid movement sequences. Journal of Experimental Psychology: Human Perception and Performance, 9, 86-102.

Rubinstein, J. S., Meyer, D. E., \& Evans, J. E. (2001). Executive control of cognitive processes in task switching. Journal of Experimental Psychology: Human Perception and Performance, 27, 763-797.

Ruthruff, E., Remington, R. W., \& Johnston, J. C. (2001). Switching between simple cognitive tasks: The interaction of top-down and bottom-up factors. Journal of Experimental Psychology: Human Perception and Performance, 27, 1404-1419.

Scheffers, M. K., \& Coles, M. G. H. (2000). Performance monitoring in a confusing world: Error-related brain activity, judgments of response accuracy, and types of errors. Journal of Experimental Psychology: Human Perception and Performance, 26, 141-151.

Schneider, D. W., \& Logan, G. D. (in press). Modeling task switching without switching tasks: A short-term priming account of explicitly cued performance. Journal of Experimental Psychology: General.

Sohn, M.-H., \& Anderson, J. R. (2001). Task preparation and task repetition: Two-component model of task switching. Journal of Experimental Psychology: General, 130, 764-778.

Sohn, M.-H., \& Carlson, R. (2000). Effects of repetition and foreknowledge in task-set reconfiguration. Journal of Experimental Psychology: Learning, Memory, and Cognition, 26, 1445-1460.

Townsend, J. T., \& Ashby, F. G. (1983). Stochastic modeling of elementary psychological processes. New York: Cambridge University Press.

Waszak, F., Hommel, B., \& Allport, A. (2003). Task-switching and longterm priming: Role of episodic stimulus-task bindings in task-shift costs. Cognitive Psychology, 46, 361-413.

Wylie, G. R., \& Allport, D. A. (2000). Task switching and the measurement of "switch costs." Psychological Research, 63, 212-233.

Yeung, N., \& Monsell, S. (2003a). The effects of recent practice on task switching. Journal of Experimental Psychology: Human Perception and Performance, 29, 919-936.

Yeung, N., \& Monsell, S. (2003b). Switching between tasks of unequal familiarity: The role of stimulus-attribute and response-set selection. Journal of Experimental Psychology: Human Perception and Performance, 29, 455-469.

Received July 26, 2004 Revision received November 17, 2004 Accepted November 29, 2004

\section{ORDER FORM}

Start my 2005 subscription to Journal of Experimental Psychology: Learning, Memory, and Cognition! ISSN: 0278-7393

\section{$\$ 140.00$, APA Member/AfFiliate $\$ 287.00$, Individual NoNMEMber $\$ 700.00$, InstiTution In $D C$ add $5.75 \%$ / In $M D$ add $5 \%$ sales tax Total Amount Enclosed}

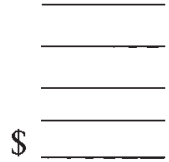

Subscription orders must be prepaid. (Subscriptions are on a calendar year basis only.) Allow 4-6 weeks for delivery of the first issue. Call for international subscription rates.

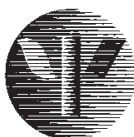

SEND THIS ORDER FORM TO:

American Psychological Association

Subscriptions

750 First Street, NE

Washington, DC 20002-4242

AMERICAN PSYCHOLOGICAL ASSOCIATION
Or call (800) 374-2721, fax (202) 336-5568

1 $336-6123$

For subscription information, e-mail:

subscriptions@apa.org
Send me a FREE Sample Issue

(3) Check enclosed (make payable to APA)

Charge my: OVISA MasterCard American Express

Cardholder Name

Card No. Exp. Date

Signature (Required for Charge)

\section{BILLING ADDRESS:}

Street

City____ State _... Zip ___ _ _ _ _

Daytime Phone

E-mail

SHIP TO:

Name

Address

City State Zip

APA Member \# $X Z M A 15$ 